\title{
Elucidating the neural mechanisms of Learning-to-Learn
}

\author{
Vishwa Goudar ${ }^{1}$, Barbara Peysakhovich ${ }^{2}$, David J. Freedman ${ }^{2,3}$ \\ Elizabeth A. Buffalo ${ }^{4,5}$, and Xiao-Jing Wang *1 \\ ${ }^{1}$ Center for Neural Science, New York University, NY, USA \\ ${ }^{2}$ Department of Neurobiology, The University of Chicago, Chicago, IL, USA \\ ${ }^{3}$ Grossman Institute for Neuroscience, Quantitative Biology and Human Behavior, The \\ University of Chicago, Chicago, IL, USA \\ ${ }^{4}$ Department of Physiology and Biophysics, University of Washington, Seattle, WA, \\ $U S A$ \\ ${ }^{5}$ Washington Primate Research Center, University of Washington, Seattle, WA, USA
}

*Corresponding Author: xjwang@nyu.edu 


\begin{abstract}
Learning-to-learn, a progressive acceleration of learning while solving a series of similar problems, represents a core process of knowledge acquisition that draws attention in both neuroscience and artificial intelligence. To investigate its underlying brain mechanism, we trained a recurrent neural network model on arbitrary sensorimotor mappings. The network displayed an exponential speedup in learning. The neural substrate of a schema emerges within a low-dimensional subspace of population activity. Its reuse in new problems facilitates learning by limiting connection weight changes. Since the population trajectory of a recurrent network produces behavior, learning is determined by the vector field changes. We propose a novel analysis of weight-driven vector field changes, which showed that novel stimuli in new problems can distort the schema representation. Weight changes eliminate such distortions and improve the invariance of the reused representations in future learning. The accumulation of such weight changes across problems underlies the learning-to-learn dynamics.
\end{abstract}




\section{Introduction}

In Psychology, Schema refers to an abstract mental representation that we deploy to interpret and respond to new experiences, and recall these experiences later from memory (Piaget 1926; Bartlett 1932). Mental schemas are thought to express knowledge garnered from past experiences (Bartlett 1932; Rumelhart 1980; Gilboa and Marlatte 2017). For example, expert physicists apply relevant physics schemas when they categorize mechanics problems based on governing physical principles (e.g. conservation of energy or newton's second law); by contrast, novice physicists who lack these schemas resort to categories based on concrete problem cues (e.g. objects in the problem or their physical configuration) (Chi et al. 1981). What is the brain mechanism of schemas, and what makes it essential for rapid learning and abstraction?

One type of schema is called "learning set". In a pioneering experiment, H. F. Harlow trained macaque monkeys on a series of stimulus-reward association problems (Harlow 1949). While keeping the task structure fixed, each problem consisted of two novel stimuli that had to be correctly mapped onto reward versus nonreward, respectively. Harlow found that the monkeys progressively improved their learning efficiency over the course of a few hundred problems, until they could learn new problems in one shot. He concluded that rather than learning each problem independently of the earlier ones, the monkeys formed an abstract learning set that they deployed to learn new problems more efficiently - they were learning-to-learn. Evidence of schema formation from prior knowledge has been demonstrated in humans (Thorndyke and Hayes-Roth 1979; Van Kesteren et al. 2013) and nonhuman animals (Steele and Morris 1999; Tse et al. 2007; Zhou et al. 2020). Moreover, converging lines of evidence derived from functional connectivity (Brodt et al. 2018; Hebscher et al. 2019), structural plasticity (Brodt et al. 2018), lesion (Tse et al. 2007), pharmacological blockade (Wang et al. 2012), and gene expression (Tse et al. 2011) studies, attribute the acceleration of learning to the rapid integration of new experiences into pre-existing schema that are encoded in the neocortex. This has motivated a neurocentric definition of a schema as a network of strongly interconnected neocortical representations that affect processing of new in- 
formation (Van Kesteren et al. 2012; Van Kesteren et al. 2013; Hebscher et al. 2019). However, these previous experiments did not elucidate how, mechanistically, a neural circuit realizes a schema and expedites learning.

Schemas are posited to emerge as an abstraction of the commonalities across previous experiences (Lewis and Durrant 2011; Gilboa and Marlatte 2017). It is the generalization of these abstract representations to novel situations that is believed to accelerate learning (Wang and Morris 2010; Preston and Eichenbaum 2013; Behrens et al. 2018). Indeed, the abstract neural representation of shared task variables has been observed across consecutively learned problems when experience on earlier problems facilitates later learning (McKenzie et al. 2014; Bernardi et al. 2020). Furthermore, the progressive improvement in learning efficiency observed by Harlow suggests that this process of abstract representation-facilitated learning undergoes progressive refinement. The structure learning hypothesis (Braun et al. 2010) equates learning to a change in the brain's internal parameters which control behavior, and posits that the progressive improvement in learning efficiency emerges with a low-dimensional task-appropriate realization of the internal parameter space. Parameter exploration within such a space is less demanding, which makes learning more efficient. Therefore, while schema formation emphasizes an abstraction of the task's structure, structure learning emphasizes learning how to efficiently use a schema to aid in generalization.

In spite of tremendous progress in machine intelligence, learning-to-learn presents a major challenge in presently available artificial systems. Machine learning studies have proposed meta-learning approaches wherein model parameters that promote rapid generalization to new problems are explicitly favored and sought (Finn et al. 2017; Wang et al. 2018). Yet, it is not known whether such mechanisms are necessary computationally or present in the brain. Can learning-to-learn arise solely from the natural dynamics of learning?

We explored this question of broad interest to brain research, cognitive science and artificial intelligence. As a behavioral paradigm we chose learning of arbitrary sensorimotor associations, which requires the learning of arbitrary mappings between sensory 
stimuli and motor consequents (Asaad et al. 1998; Fusi et al. 2007), and is essential for flexible behavior (Passingham 1995). Macaque monkeys can learn-to-learn association problems - they demonstrate an exponential decrease in the number of trials to learn each of several problems (Peysakhovich et al., Unpublished), and learn new problems within an average of 20 trials when they are well-trained (Cromer et al. 2011). Furthermore, their prefrontal cortex is causally engaged during rapid problem learning. Prefrontal neurons represent task stimuli and responses during visuomotor association trials (Asaad et al. 1998; Cromer et al. 2011). Prefrontal lesions produce substantial visuomotor association learning deficits (Petrides 1985; Passingham 1995; Bussey et al. 2002). We sought to understand whether and how a sensorimotor association schema may be encoded by these prefrontal representations, how it is applied to new problems, and how its usage is refined to improve learning efficiency.

Towards this end, we trained and analyzed a recurrent neural network (RNN) model of prefrontal computation and learning. We found that RNNs trained on a series of sensorimotor association problems exhibit robust learning-to-learn despite the absence of meta-learning: the number of trials to learn a problem decays exponentially with the number of previously learned problems. We analyzed the population activity of the RNN's units via subspace decomposition to uncover population-level latent variable representations (Stringer et al. 2019; Zhou et al. 2020), and manifold perturbations to study the causal relationship between learning efficiency and the reuse of existing population representations to learn (Sadtler et al. 2014). The analyses revealed that the model develops neural correlates of the task's schema - a low-dimensional neural manifold that represents shared task variables in an abstract form across problems. Its reuse avoids the formation of new representations while learning problems which considerably accelerates learning by limiting the connection weight changes required to learn them. We introduce a novel measure relating these weight modifications to population activity changes, which we term the weight-driven vector field change. It showed that the reused representations are not entirely invariant across problems. Instead mapping new stimuli can modify the reused representations in undesirable ways. Connection weight changes are primarily recruited to prevent such modifications. Moreover, the weight changes in 
115 early problems improve the invariance of the reused representations, which limits the 116 degree to which they would be modified in the future, which further accelerates learning.

${ }_{117}$ The accumulation of such improvements over a series of problems supports structure ${ }_{118}$ learning and promotes learning-to-learn. 


\section{Results}

\section{Trained neural network models learn-to-learn without meta-learning}

We evaluated whether an RNN model could learn-to-learn delayed sensorimotor association problems. In each problem, the model learned a unique mapping between a pair of sensory stimuli (e.g. images) and a pair of motor responses (Fig. 1a). Each trial began with a 0.5 seconds sample epoch, when a sensory stimulus was presented together with a fixation cue, and the model was required to maintain fixation. A 1 second delay epoch followed, when the model had to continue fixation in the absence of the sample stimulus. The trial concluded with a 0.5 second choice epoch signalled by removal of the fixation cue, when the model had to report its choice of an appropriate motor response. The two sample stimuli in each problem were randomly generated. The model was composed of a population of recurrently (or laterally) connected firing rate units that received eleven inputs, one signalling fixation and ten signalling features of a sample stimulus (Fig. 1 $1 \mathrm{~b}$ ). Such stimulus representations are consistent with a recent finding that visual objects are represented in the monkey inferotemporal cortex by a feature-based topographic map (Bao et al. 2020). The model is also consistent with lesion studies which demonstrate the causal involvement of inferotemporal-prefrontal connections in visuomotor learning and retention (Eacott and Gaffan 1992; Bussey et al. 2002). Response choices were read out from the population's activity by three output units that represented fixation, motor response 1 , or motor response 2 .

The model was trained on a problem one trial at a time. Its parameters were adjusted at the end of each trial to minimize the errors in its output responses, until the output responses achieved criterion accuracy (see Methods). The model was then transitioned to a new problem (Fig. 1c). Crucially, training was performed without an explicit metalearning objective. A network trained on a series of delayed sensorimotor association problems learned-to-learn (Fig. 1d). The network required a few thousand trials to learn the first problem, but only a few hundred to learn the second one. Its trials-to- 
criterion progressively decreased over the next few hundred problems, plateauing at an average of 20 trials per problem. This decrease was well-fit by a decaying exponential function, which closely matched a 30-problem moving average of the network's trials-tocriterion. This performance is commensurate with learning-to-learn in macaque monkeys, which exhibit an exponential decrease in their trials-to-criterion when trained on a series of association problems (Peysakhovich et al., Unpublished), and demonstrate learning within 15-20 trials when well-trained (Cromer et al. 2011). The fit's parameters quantify the network's learning-to-learn performance: the time constant measures how quickly it learns-to-learn, and the learning efficiency asymptote measures its trials-to-criterion plateau. We note that while naive monkeys undergo behavioral shaping on the desired response set before they are introduced to the task, a naive network's learning efficiency on the first problem reflects learning both to generate basic responses, and the specifics of the problem. To avoid this confound related to learning the response set, we quantified the network's learning-to-learn performance starting with the second problem.

We tested the robustness of these results by similarly training 30 independently initialized networks. Across these networks, the learning-to-learn time constants and asymptotes were limited to a narrow range (Fig. 1e; time constant mean $=47.52$, std. $\operatorname{dev} .=26.22$; asymptote mean $=21.33$, std. $\operatorname{dev}=3.85)$. We further tested the model over a range of hyper-parameter settings (f-I transfer functions, learning rates, weight and firing rate regularization levels), and observed robust learning-to-learn across all conditions (Supplementary Fig. 11). Taken together, these results demonstrate that networks trained on a series of delayed sensorimotor association problems robustly learnto-learn, despite the absence of an explicit meta-learning objective.

\section{Abstracted neural manifold governs the task's schema and drives output responses}

The activity of a recurrently connected population of $N$ units co-evolves over the duration of a trial, forming a trajectory in an $N$-dimensional population state space (Fig. 2a, 
top). When a problem is learned, the network responds to each sample stimulus with a trajectory that appropriately subserves stimulus integration, decision making, working memory maintenance, and fixation/motor response choice. We demixed (Kobak et al. 2016; see Methods) population trajectories from consecutively learned problems to identify shared representations, if any, of the latent variables that support these computations. This procedure decomposed the trajectories into components embedded within two non-overlapping subspaces of the population state space (Fig. 2a, middle): Decision representations embedded within the decision subspace revealed similarities between trajectories that shared target responses; stimulus representations embedded within the stimulus subspace varied in a problem- and sample stimulus-dependent manner. We further decomposed the two decision representations in each problem into a mean decision representation, where the mean was taken over both decision representations (Fig. 2a, bottom left), and residual decision representations given by subtracting out this mean from the two decision representations (Fig. 2a, bottom right).

Decomposing the trajectories from the first 50 consecutively learned problems in this manner revealed a low-dimensional shared decision subspace (mean $=2.36$ dimensions; std. dev. $=0.18$ dimensions across 10 networks), whose constituent decision representations explained most of the variance in population activity across problems (mean $=88.54 \%$; std. dev. $=3.16 \%$ across 10 networks). Furthermore, the mean decision representations lay close to each other in state space, forming a shared manifold across problems (Fig. 2b, left). The residual decision representations consistently encoded the decision and choice of either response across problems, thus forming a shared manifold for each decision (Fig. 2b, center). The persistence of a low-dimensional shared manifold which explains a majority of the population's variance across problems demonstrates a strong abstraction of the shared task variables that it encodes. It bears mentioning that the model retains and reuses this manifold across problems, despite changes in the stimulus set and the weight change-induced change in network dynamics that transpires while learning. In contrast, the stimulus representations (Fig. 2b, right) were higher dimensional $($ mean $=7.98$ dimensions; std. dev. $=1.48$ dimensions across 10 networks), but explained a small proportion of the population variance. Interestingly, the distribu- 
tion of neural activity in population state space at the beginning and end of problem learning closely resembled each other (Supplementary Fig. 2), suggesting that the model even reuses pre-established representations when responding to novel sample stimuli.

Next, we examined the relative contribution of these components to the output responses by measuring the net current from each component to the choice outputs (Fig. 2c). During trials where response 1 was chosen (mapping 1 trials), residual decision representations excited the response 1 output unit and inhibited the response 2 output unit, particularly within the choice epoch (Fig. 2d, center). During mapping 2 trials, these representations had the opposite effect. In contrast, the mean decision representations inhibited both response choices throughout the sample and delay epochs, but not the choice epoch (Fig. 2d, left). This was essential to preventing premature response choice initiation during the delay epoch (Fig. 2d, center). The contribution of the stimulus representations to response selection was negligible throughout the trial (Fig. 2d, right). Quantitatively similar results were obtained for all consecutively learned 50-problem groups in all the networks that we tested. These results demonstrate that the decision manifold constitutes the neural correlates of the task's schema — it represents the shared temporal (mean decision) and 2-alternative (residual decision) structure of the task in an abstract form, and thereby reflects knowledge abstracted from past experiences. This predicts that the decision manifold facilitates generalization of the task structure.

\section{Schematic manifold embodies a representational scaffold that facilitates learning}

We have shown that the schematic decision manifold is reused by, or scaffolds (Rumelhart and Norman 1976; Anderson et al. 1978; Thorndyke and Hayes-Roth 1979), the learned representations in subsequent problems. Moreover, this reuse is accompanied by a stark improvement in learning efficiency between the first problem and subsequent ones (Fig. 1d). To establish whether the reuse of the decision manifold plays a causal role in improving learning efficiency, we compared the learning efficiency of networks that were 
barred from reusing the existing decision manifold to control output responses in new problems, with networks that were allowed to do so. This method has been applied in brain-computer interface (BCI) studies to establish a causal link between monkeys' ability to rapidly adapt to BCI readout perturbations and their reuse of existing motor cortical representations to modulate the perturbed readouts (Sadtler et al. 2014).

In our model, this intervention relies on the concept of a readout subspace. Population activity modulates an output unit's response, only when the sum of the excitatory and inhibitory postsynaptic currents it produces at the unit are non-zero (output-potent activity, Kaufman et al. 2014). Since these currents depend of the model's output connection weights, the output weights constrain the set of population activity levels which can modulate output unit responses. This set defines the readout subspace of population state space. Our observation that population representations within the decision subspace predominantly modulate output responses implies that the decision subspace strong overlaps with the readout subspace. It follows that the elimination of this overlap, by appropriately altering the readout subspace, would force the development of new decision representations to modulate the output responses. This would impair the effectiveness of the representational scaffold provided by the pre-existing decision manifold in composing the learned trajectories. The observation of a concurrent learning deficit would establish a causal link between the representational scaffold and accelerated learning. For this causal intervention as well as its controls, we started by training a naive network on a single problem so that it appropriately developed overlapping readout and decision subspaces (Fig. 3a).

In the frozen readout condition, we then trained the network on its second problem while freezing (or preventing changes to) the output weights (Fig. 3b, top right). This helped assess whether freezing the output weights adversely affects learning efficiency. Results showed that networks exhibited a substantial improvement in learning efficiency from the first problem to the second despite frozen readouts (Fig. 3c).

In the stimulus-to-stimulus $(\mathrm{S} \rightarrow \mathrm{S})$ manifold perturbation condition, we perturbed the output weights such that the overlap between the readout and stimulus subspaces 
was altered, but the overlap between the readout and decision subspaces was not (Fig. 3b, bottom left; see Methods). We then trained the network on its second problem with frozen output weights, which prevented the training procedure from re-aligning the readout subspace with the stimulus subspace. In these networks as well, we found a substantial improvement in the learning efficiency from the first problem to the second (Fig. 3c).

Finally, in the decision-to-stimulus $(\mathrm{D} \rightarrow \mathrm{S})$ manifold perturbation condition, we perturbed the output weights such that the readout subspace overlapped exclusively with the stimulus subspace (Fig. 3b bottom right). We then trained the network on its second problem with frozen output weights. This condition eliminates any overlap between the readout and decision subspaces, and compels the formation of new decision representations within the original stimulus subspace. In contrast to networks with frozen readouts and $\mathrm{S} \rightarrow \mathrm{S}$ manifold perturbations, we found that the learning efficiency of these networks was strongly impaired - they learned as slowly as naive networks learning their first problem (Fig. 3c). Taken together, these results demonstrate that $\mathrm{D} \rightarrow \mathrm{S}$ manifold perturbations adversely affected learning performance because the reuse of the decision manifold was impeded, and not because this was achieved by perturbing and freezing the output weights. This confirms that the schematic decision manifold forms a representational scaffold that facilitates the transfer of prior knowledge regarding the task's structure to new problems, and expedites learning in the process.

\section{Representational reuse and synaptic plasticity differentially con- tribute to learning}

We have shown that the reuse of existing representations to learn problems improves learning efficiency. However, learning produces fairly large changes in population activity to mediate the necessary output response corrections (Supplementary Fig. 6b). How does the emergence of such sizeable changes benefit from the reuse of existing representations? And how do the contributions of this reuse compare to those of the 
plasticity-induced connection weight changes? To answer these questions, we analyzed the activity changes between the beginning and end of a problem.

The neural population responds to a novel sample stimulus with a pre-learning trajectory in population state space (Fig. 4 b left, dashed curve). This trajectory evolves through time due to the repetition of the following process (equation (2)): The inputs and population activity at time $t-1$ generate postsynaptic currents subject to the network's recurrent and input connection weights; these currents are integrated by the network units, which advances their activity from $\boldsymbol{r}_{t-1}^{\prime}$ to $\boldsymbol{r}_{\boldsymbol{t}}^{\prime}$ (Fig. 4a, top). In state space, this instantaneous advance in the population state is represented by a vector originating at $r_{t-1}^{\prime}$. Note that the direction and magnitude of this advance is state-dependent - it depends on the activity levels of the population's units, i.e. the population state, at time $t-1$. The temporal sequence of these vectors guides the evolution of the population's activity between its initial $(\boldsymbol{r}(t=0))$ and final $\left(\boldsymbol{r}_{\boldsymbol{T}}^{\prime}\right)$ states (Fig. 4 b, top center, light blue arrows). Importantly, these state-dependent vectors constitute a vector field (Strogatz 2016; Vyas et al. 2020) that spans the entire state space and describes the network's dynamics. Note that the vector field produced by a network depends on its connection weights.

When the problem is learned, the population activity traverses a learned trajectory (Fig. 4b left, solid curve) comprised of learned population states, $\boldsymbol{r}_{\boldsymbol{t}}$. Since the connection weights at the end of a problem are given by the sum of the pre-learning weights and the plasticity-induced weight changes, this learned trajectory is governed by the sum of the pre-learning vector field and the changes in this field caused by the weight changes. Consequently, changes in population activity from the pre-learning to the learned trajectory are also governed by these two factors. The change in population activity from a pre-learning to a learned state at time $t, z_{t}$, is represented by a vector in state space from the former to the latter (Fig. $4 \mathrm{~b}$, top center, solid lime-green arrows). It stems from three components (Fig. $4 \mathrm{~b}$, center; see Methods). The first is the activity change up to time $t-1\left(z_{t-1}\right)$. The remaining components individually measure the contributions of the pre-learning vector field (i.e. the reuse of existing representations) and the plasticity-induced changes in the vector field. 
Setting aside the effect of weight changes for a moment, consider the network's prelearning vector field at the learned state $\boldsymbol{r}_{t-\mathbf{1}}$ versus at the pre-learning state $\boldsymbol{r}_{\boldsymbol{t - 1} \mathbf{1}}^{\prime}$. Due to its state-dependence, the pre-learning vector field may advance population activity quite differently from one state versus the other. In this event, the activity difference between the pre-learning and learned states will change between times $t-1\left(\boldsymbol{z}_{t-1}\right)$ and $t\left(\boldsymbol{z}_{\boldsymbol{t}}\right)$, by an amount $\boldsymbol{\Delta} \boldsymbol{F i e l} \boldsymbol{d}_{\boldsymbol{s}, \boldsymbol{t}}$. In state space, this change is reflected by the vector difference (Fig. $4 \mathrm{~b}$, bottom right, pink arrow) between the pre-learning vector field at the two states (solid light blue arrows). This component is referred to as the state-driven vector field change (abbreviated as SdVFC; equation (6)), and it solely depends on the pre-learning vector field (i.e. on existing representations).

The third component, $\Delta \boldsymbol{F i e l} \boldsymbol{d}_{\boldsymbol{w}, t}$, is determined by the connection weight changes which alter the net postsynaptic currents into the population, thereby altering how the population activity advances between times $t-1$ and $t$ (Fig. 4a, bottom). In state space, this translates to a change in the vector field along the learned trajectory (Fig. $4 \mathrm{~b}$, top right, orange arrow). This component is referred to as the weight-driven vector field change (abbreviated as WdVFC; equation (7)). The sum of the two vector field change components (WdVFC and SdVFC) produces an incremental change in population activity $\left(\Delta \boldsymbol{z}_{t}\right)$ between times $t-1$ and $t$ (Fig. $4 \mathrm{~b}$, center, dark-green arrow; equations (4)-(5)).

Measurements revealed a substantial difference between the magnitudes of the activity changes $\left(\boldsymbol{z}_{\boldsymbol{t}}\right.$; Supplementary Fig. 6b) and the activity change increments $\left(\Delta \boldsymbol{z}_{\boldsymbol{t}}\right.$; Fig. 5a). That is, large population activity changes emerge from an accumulation of relatively small change increments generated throughout the trial. We further assessed the relative contribution of the WdVFC and SdVFC to the activity change increments by decomposing them (see Methods) into their respective components in the direction of the activity change increments $\left(\Delta \boldsymbol{z}_{\|}\right.$- parallel component) and orthogonal to them ( $\Delta \boldsymbol{z}_{\perp}$ - orthogonal component; Fig. 4 b, right).

A key observation was that the SdVFC's $\left(\boldsymbol{\Delta}\right.$ Field $\left.\boldsymbol{s}_{\boldsymbol{s}}\right)$ parallel component is much larger in magnitude than the WdVFC's $\left(\Delta \boldsymbol{F i e l d}_{\boldsymbol{w}}\right)$ parallel component (Fig. 5a, green 
bars). Therefore, the network's pre-learning vector field, which governs the SdVFC, is primarily responsible for the population activity changes. Dimensionality measurements of these parallel components revealed that they are low-dimensional not only in individual problems, but also across a group of problems (Fig. 5b). This is consistent with the structure learning hypothesis (Braun et al. 2010), which posits that efficient learning relies on changing behavior via parametric changes within a low-dimensional internal parameter space of the brain. Our results suggest that this low-dimensional internal parameter space corresponds to a low-dimensional subspace of neural population activity, which constrains how population activity may change when learning a problem.

Measurements also showed that the WdVFC's orthogonal component is much larger in magnitude than its parallel component, and that it is equal in magnitude but opposite in direction to its state-driven counterpart and nullifies it as a result (Fig. 5a, pink bars). These orthogonal components of the WdVFC and SdVFC are also low-dimensional on individual problems, but are high-dimensional across a group of problems (Fig. 5b). Moreover, they largely span directions that the existing representations do not typically covary along (Supplementary Fig. 7a). These results imply that novel sample stimuli interact with the existing representations when mapped onto them, in a manner that elicits uncharacteristic population responses. They also reveal that the existing representations can be sensitive (i.e. not entirely invariant) to the sample stimuli that are mapped onto them. The WdVFC is primarily meant to impede such interactions, thereby preventing changes to the existing representations.

To summarize, our analysis of the population activity changes between the start and end of problem learning revealed that: (i) large changes emerge over the trial timecourse from the accumulation of a sequence of small local changes along the learned trajectory; (ii) these changes are low-dimensional and stem primarily from reusing the network's pre-learning vector field to shape the learned trajectory, thus elucidating the relative contribution of representational reuse to learning; (iii) the pre-existing representations are not entirely invariant to having novel sample stimuli mapped onto them, and can undergo uncharacteristic modifications in the process. Connection weight changes largely prevent such modifications. 


\section{Magnitude of recurrent weight changes determines learning ef- ficiency}

Next, we examined why learning efficiency is enhanced by representational reuse, by exploring how learning efficiency is impacted by the connection weight changes. Before we could do so, it was important to evaluate the relative contribution of input versus recurrent weight changes to learning. In supplementary note 1.1, we show that the model learns via recurrent weight changes - these changes predominantly determine the WdVFC - as it is more efficient to do so. Moreover, measurements showed that the magnitude of recurrent weight changes in a problem largely explains the number of trials expended in learning it (Fig. 6a, left; coefficient of determination $R^{2}=0.7$ ). This relationship was robustly observed across all 10 networks that were tested (Fig. 6a, right).

In light of this observation and the exponential decrease in the trials-to-criterion across problems, we hypothesized that the magnitude of recurrent weight changes should also decrease exponentially over the sequence of learned problems. We further posited that the recurrent weight change magnitudes should be proportional to the postsynaptic current change and the WdVFC magnitudes, since these three quantities are directly related to each other. Consequently, we expected that the magnitudes of the postsynaptic current changes and the WdVFC would also decrease exponentially. Figure $6 \mathrm{~b}$ confirms that the magnitude of these three quantities decreases exponentially as a function of the number of previously learned problems. Therefore, the progressive improvement in the model's learning efficiency is explained by a similar decrease in the magnitudes of the recurrent weight changes and WdVFC required to learn problems.

We can now explain why the reuse of existing representations markedly improves learning efficiency (Fig. 3). Networks with $\mathrm{D} \rightarrow \mathrm{S}$ manifold perturbations are compelled to develop new representations of the task's structure beyond the original decision subspace, and aligned with their perturbed readout subspace (Fig. 3b, bottom right). In other words, the structure and location, in state space, of the target trajectories are 
largely constrained by the arbitrarily altered output weights. However, the vector field along such an arbitrarily constrained target trajectory is most likely misaligned relative to the vector field required to support it (Supplementary Fig. 5a, right, dark versus light blue arrows along learned trajectory). Consequently, it is unlikely to roughly advance population activity along the target trajectory, as it does in unperturbed networks (Supplementary Fig. 5a, left). Measurements comparing the magnitude of the WdVFC in unperturbed and perturbed networks confirms that the vector field in perturbed networks undergo drastic re-organization in comparison to unperturbed networks (Supplementary Fig. 5b, right), so that they may shape the trajectories that will re-encode the task's structure (large orange arrows, Supplementary Fig. 5a). This explains the impairment in learning efficiency following $\mathrm{D} \rightarrow \mathrm{S}$ manifold perturbations and demonstrates the merits of learning via representational reuse - it is this reuse of existing representations that limits the requisite weight changes (Supplementary Fig. 5b, left), and thereby improves the learning efficiency.

In supplementary note 1.2, we explore the interactions between stimulus and decision representations during trial performance and learning. We found that the stimulus and decision representations exhibit reciprocal interactions to sustain each other through the trial, and that the WdVFC largely prevents uncharacteristic changes in the existing stimulus representations (rather than existing decision representations). In addition, we assessed whether the WdVFC is modulated more strongly by pre-synaptic population activity in the stimulus or decision subspace. A comparison of their approximate contributions to the WdVFC revealed that it relies almost entirely on decision representations (Fig. 6c, Supplementary Fig. 6d). This is likely due the the fact that the decision representations are larger in magnitude than the stimulus representations. These results reveal a second form of representational scaffolding, wherein the decision representations scaffold the formation of the WdVFC. 


\section{Accumulation of weight changes across problems progressively improves learning efficiency}

In agreement with Harlow's learning-to-learn experiments, our model exhibits a progressive improvement in learning efficiency spanning a few hundred problems (Fig. 1). This improvement is explained by a progressive decrease in the magnitude of the weight changes and WdVFC per problem (Fig. 6a-b). Since the WdVFC in a problem primarily prevents distortions to existing representations during learning (Fig. 5a), a progressive decrease in its magnitude amounts to a progressive improvement in the invariance of the existing representations to having novel stimuli mapped onto them. However, the source of this improvement is yet undetermined: what causes it in the absence of an explicit meta-learning mechanism, and how does the network's accumulation of learning experience over problems relate to its emergence? We hypothesized that the accumulation of weight changes over earlier problems facilitates learning in future problems. That is, weight changes elicited while learning problems $p-k$ (for $1 \leq k \leq p-2$ ) cumulatively alter the vector field such that they suppress the WdVFC required to learn problem $p$ (Supplementary Fig. 8a, top; see Methods). More generally, as problems are learned, their respective WdVFCs accumulate to produce a cumulative vector field change (abbreviated as CVFC) which suppresses the WdVFC required to learn subsequent problems. This progressively improves representational invariance and thereby accelerates learning.

To test this hypothesis, for each problem $p$, we measured the magnitudes of its WdVFC plus the CVFC along its learned trajectory due to the accumulation of weight changes over the sequence of problems that precede it, i.e. from problem $p-1$ (relative problem -1) to problem 2 (relative problem $2-p$ ). Figure ta summarizes these measurements across many problems $p$ grouped by their learning-to-learn stage, i.e. the number of problems they are preceded by. Here, we focused on the magnitude along each problem's orthogonal WdVFC component $\left(\Delta \boldsymbol{z}_{\perp}\right)$, because it dominates the total WdVFC in problems at each learning-to-learn stage (Supplementary Fig. 7b). The results indeed show that at each stage, learning earlier problems cumulatively suppresses the WdVFC required in subsequent problems. We further found that this is predominantly due to an 
accumulation of recurrent weight changes (Supplementary Fig. 7c). These findings confirmed our hypothesis - the accumulation of weight changes over problems progressively improves representational invariance and therefore learning efficiency. Moreover, they imply that the cumulative change along the orthogonal WdVFC component of problems imposes a learning efficiency bottleneck.

Surprisingly, even though the network expends many more trials on learning early problems, the approximate linearity of the curves in figure 7 a indicates that early- and late-learned problems produce similar-sized contributions to the CVFCs. Indeed, measurements showed that the per-trial CVFC contributions by late-learned problems are larger than those by early-learned problems (Supplementary Fig. 9a). This demonstrates that with experience, the model learns to contribute to the learning efficiency of future problems in an increasingly efficient manner.

Figure 7a further demonstrates that the WdVFC in a problem depends on its net suppression by the preceding problems, i.e. the sum of the suppressive CVFC contributions (and enhancing CVFC contributions when they increase the requisite WdVFC) by the weight changes in each preceding problem going back to problem 2 (Supplementary Fig. 8b, left; see Methods). A larger net suppression produces a smaller WdVFC. Since the WdVFC decays exponentially with the number of preceding problems (Fig. 6b), we posited that the net suppression must similarly increase with it. Measurements of the net suppression along the orthogonal and parallel WdVFC components of problems confirmed this (Fig. 7b). The net suppression mirrors the exponential decay in the WdVFC (see Methods) - it rapidly increases across problems at the early stages of learning-tolearn, which produces a rapid decrease in their WdVFCs; it gradually plateaus for later problems, which explains the plateauing of their WdVFCs. The results also showed that the net suppression is weaker along the orthogonal components than along the parallel components, which explains why the learning efficiency bottleneck develops along the orthogonal components.

Figure $7 \mathrm{~b}$ also revealed that the net suppression of a problem's WdVFC is not linearly related to the number of problems that precede it. This indicates that the rate at which 
the CVFC contributions suppress a problem's WdVFC depends on its learning-to-learn stage. We reasoned that slow (quick) suppression must be due to smaller (larger) contributions by the weight changes in preceding problems. Therefore, we expected that the sum of the magnitudes of these contributions would be small (large) for problems whose WdVFC is suppressed slowly (quickly), and the progression of this sum over the learningto-learn stages would resemble the net suppression magnitudes (Fig. 7b). Instead, we found that the sum increases in a largely linear fashion for the CVFC contributions along both the parallel and orthogonal WdVFC components (Supplementary Fig. 9b). This indicated that $(i)$ the WdVFC of problems at different learning-to-learn stages are altered to a similar extent by weight changes in the problems that precede them, and (ii) the sum of the magnitudes of these CVFC contributions reflects the number of problems that they accumulate over, but not the differences in their suppression rates. Taken together with the results in figure $7 \mathrm{~b}$, this reveals a surprising result: although the CVFC contributions are similar-sized, their suppressive effect on a future problem's WdVFC depends on its learning-to-learn stage.

Interestingly, along both the parallel and orthogonal WdVFC components, we observed that the sum of contribution magnitudes (Supplementary Fig. 9b) is orders of magnitude larger than the net suppression magnitudes (Fig. 7fb). In other words, relatively large CVFC contributions from earlier problems cumulatively suppress an ensuing problem's WdVFC by relatively small amounts. Based on this observation, we concluded that the effect of weight changes in individual problems on a given future problem's WdVFC are largely inconsistent with each other; some CVFC contributions suppress the problem's WdVFC while others enhance it (Supplementary Fig. 8b, right). In fact, the ratio of the net suppression magnitude to the sum of contribution magnitudes quantifies this consistency; a value of 1 would indicate that the problem's WdVFC is suppressed by the weight changes in each of its preceding problems, and a value of 0 would indicate that the CVFC contributions are maximally inconsistent - the enhancing and suppressing contributions nullify each other resulting in no net change. For problems at all learning-to-learn stages, we found that this ratio was closer to 0 (Fig. $7 \mathrm{~d}$ ).

These result depict the suppressive effect of the accumulating weight changes on 
each future problem's WdVFC as a stochastic process - CVFC contributions by individual problems stochastically enhance or suppress the future problem's WdVFC. However, they collectively exhibit a weak bias towards consistently suppressing it (values in Fig. 7d are above zero). The cumulative effect of this weakly suppressive bias is a small yet significant suppression of the WdVFC. We illustrate this process along the orthogonal WdVFC component of an example problem. Since the orthogonal WdVFC components in a problem are roughly one-dimensional (Fig. 5b), the stochastic process is one-dimensional as well. The individual CVFC contributions by earlier problems towards suppressing the WdVFC along this dimension in the example problem (grey curve) fluctuate between positive (suppressing) and negative (enhancing) values (Fig. 7c). However, due to their weakly suppressive bias, these contributions cumulatively produce a large net suppression of the problem's WdVFC (black curve).

Figure 7d further shows that the CVFC contributions are more inconsistent along the orthogonal WdVFC components than along the parallel components, indicating that their suppressive bias is weaker along the orthogonal components. This explains the weaker net suppression along the orthogonal components (Fig. 7b), and why it imposes a learning efficiency bottleneck. Crucially, figure 7d demonstrates that the WdVFC of problems at different learning-to-learn stages are suppressed at different rates due to differences in the consistently with which the weight changes in the preceding problems suppress them (Supplementary Fig. 8b). That is, the exponential decay in the WdVFC magnitudes stems from a modulation of the suppressive bias in the CVFC contributions. The exponential decay in the WdVFC magnitudes is largely caused by an exponential decay in magnitude of their orthogonal components (Supplementary Fig. 7b). The bias in the CVFC contributions to suppress the orthogonal WdVFC component in early stage problems rapidly increases (Fig. Ad). This rapidly increases their net suppression (Fig. 7b), which rapidly decreases the WdVFC required to learn them. Subsequent problems follow a more prolonged accumulation of weight changes (because they are preceded by more problems), albeit with a weakened bias to suppress their orthogonal WdVFC components. This results in the plateauing of their net suppression, and therefore of their WdVFCs. 
To summarize, our results identify a novel neural mechanism of accumulating learning experience to progressively improve learning efficiency, despite the absence of a metalearning mechanism. It relies on the accumulation of connection weight changes over learned problems to suppress the WdVFC required to learn subsequent problems and thus accelerate their learning. The model progressively accelerates learning via, $(i)$ a gradual improvement in the efficiency with which weight changes contribute to the suppression of the WdVFC in future problems (Fig. 7b), and (ii) a modulation of how consistently suppressive these contributions are (Fig. 7e). Moreover, the fact that the WdVFC primarily prevents uncharacteristic representational changes from developing when novel sample stimuli are mapped onto existing representations (Fig. 5) helps elucidate the objective of this learning-to-learn mechanism: the accumulation of weight changes over early problems improves the invariance of the existing representations to having novel sample stimuli mapped onto them. This refines the model's ability to learn via representational reuse and elicits learning-to-learn. 


\section{Discussion}

New information is far easier to learn when it is contextualized by prior knowledge. This process is thought to be facilitated by the instantiation of schemas (Rumelhart 1980; Gilboa and Marlatte 2017), which are hypothesized to correspond to neocortically encoded knowledge structures. Learning-to-learn is a constructive consequence of the reciprocal influence between learning and schema tuning, whereby schema instantiation facilitates learning, and the assimilation of learned information into the schema improves its ability to facilitate future learning. We trained an RNN model on a series of sensorimotor mapping problems to identify the neural substrate of a sensorimotor mapping schema and to study this reciprocal process.

We found that learning-to-learn is a process with three timescales (Fig. 8). The fastest timescale governs the evolution of population activity over a single trial. Subspace decomposition of this activity showed that it encodes three latent variables. First, a mean decision component which is analogous to the condition-independent component identified in prefrontal and motor cortical activity (Kaufman et al. 2016; Kobak et al. 2016) - it encodes temporal aspects of the task in a trial-condition invariant manner, and explains most of the variance in population activity. Second, a residual decision component that encodes decisions and response choices. And third, a problem stimulus representation. The first two components collectively constitute low-dimensional decision representations that control fixation and response choices.

We also found that these decision representations are shared across problems in an abstract form: the model reuses them to contextualize its neural and output responses to new sample stimuli, and to generalize from previous solutions to newer ones. Analysis of the model's learning with a manifold perturbation intervention showed that this reuse of the decision representations causes a stark improvement in learning efficiency. These results demonstrate that the network not only abstracts commonalities across problems, but also exploits them to facilitate learning (Braun et al. 2010; Tenenbaum et al. 2011; Gilboa and Marlatte 2017). Therefore, the abstract decision representations constitute 
the neural basis of a sensorimotor mapping schema (Lewis and Durrant 2011; Gilboa and Marlatte 2017). It is noteworthy that the abstraction of task variable- and task structure-encoding neural representations and their reuse in consecutively learned association problems has indeed been observed in the prefrontal cortex and hippocampus (McKenzie et al. 2014; Bernardi et al. 2020; Zhou et al. 2020).

The intermediate timescale governs the process of learning, and spans the trials between the beginning and end of learning a single problem (Fig. 8). We studied learning with a novel measure of how connection weight changes (which model the effects of long-term synaptic plasticity or LTP) influence population activity in an RNN - the weight-driven vector field change. Our results demonstrated that this measure is more informative and accurate at assessing the effects of the connection weight changes, than direct measurements of the weight changes: (i) it dissociates the contributions of the changes in different sets of connection weights more accurately than directly comparing their magnitudes; (ii) its assessments are more interpretable as they directly relate to the population activity; and (iii) it isolates the contributions of the initial weights and the weight changes to the learning-induced changes in population activity. For these reasons, these techniques contribute to a growing set of methods that aim to overcome the challenges of interpretability and explainability in RNNs (Sussillo and Barak 2013; Dubreuil et al. 2021), which hinder their adoption in neuroscience. In our analysis, these techniques were instrumental in identifying ( $i$ ) why reusing existing representations improves learning efficiency, (ii) the relative contributions of this reuse versus the connection weight changes to learning, and (iii) the mechanism underlying learning-to-learn.

Our analysis of the change in population activity that emerges over the timespan of learning identified two forms of schematic scaffolding. First, the reuse of existing schematic representations is primarily responsible for these activity changes. The reuse avoids the formation of new task-encoding representations, which substantially reduces the weight changes required to learn a problem. This dramatically accelerates its learning. Second, the WdVFC is largely modulated by the schematic representations. Moreover, the primary role of the WdVFC is to prevent the unwarranted changes to existing 
representations which develop when novel sample stimuli are mapped onto and interact with them.

In the training RNN framework, the network is initialized with random weights, as a blank slate. Therefore, it is not so reasonable to relate the use of a learning algorithm to a known biological plasticity rule. Importantly, our findings regarding the benefits of representational reuse do not directly depend on the learning algorithm we used, and may well be conserved under biologically plausible learning rules. Nevertheless, since our analysis techniques are independent of the underlying learning rules, they offer an approach to study learning and the properties of schema formation and reuse in models with biologically plausible learning rules. Our model further assumes that following schema formation, new problems continue to be learned via LTP. Indeed, rapid learning of novel schema-consistent paired associates was found to be prefrontal NMDA-receptor dependent in rodents (Wang et al. 2012), suggesting that Hebbian neocortical synaptic plasticity is likely involved in schema-facilitated learning. However, the role of other forms of plasticity, such as intrinsic (Sehgal et al. 2013) and behavioral timescale (Bittner et al. 2017) plasticity, has not been experimentally precluded. Further computational and experimental studies are required to determine their relative roles in this process.

At the slowest timescale, several problems are learned in succession with progressively improving efficiency, until asymptotic learning efficiency is realized (Fig. 8). This is the timescale of learning-to-learn. We showed that, consistent with macaque monkeys' behavior (Peysakhovich et al., Unpublished; Cromer et al. 2011), our model's trials-tocriterion performance is well-characterized by a decaying exponential function, which asymptotes at roughly 20 trials per problem. Consequently, our model suggests that learning-to-learn can emerge in animal models in the absence of explicit meta-learning. However, the brain may adopt meta-learning (Doya 2002; Fusi et al. 2007; Abraham 2008) or model-based learning (Behrens et al. 2018; Wang et al. 2018) mechanisms to facilitate learning. Further study is required to identify their neural substrates, and evaluate their role in learning-to-learn.

We have identified a novel mechanism for learning-to-learn, which relies on the accu- 
mulation of weight changes over learned problems to progressively improve the invariance of the existing representations to being reused with novel sample stimuli. An increase in this invariance suppresses the WdVFCs required to learn new problems which accelerates their learning. Interestingly, we found that these cumulative improvements are stochastic in nature - the exponential improvement in learning efficiency stems from a modulation of the bias in this stochastic process to suppress the WdVFCs in future problems.

We also found evidence in support of the structure learning hypothesis, which posits that improvements in learning efficiency are achieved by restricting the extent of parametric exploration while learning a problem (Braun et al. 2010). It has been suggested that a network's connection weights may comprise the parameter space that controls behavioral exploration, and learning efficiency improvements emerge from the progressively restriction of weight changes to a low-dimensional subspace of the space of connection weights - the control space. Instead, we found that the model learns new problems via low-dimensional changes in population activity. That is, the parametric control space is comprised of a low-dimensional subspace of the population state space. Furthermore, connection weight changes primarily restrict the activity changes to this control space rather than generate them. Learning-to-learn derives from a progressive improvement in the model's ability to autonomously (i.e. without the support of connection weight changes) restrict its changes to this control space when novel stimuli are mapped onto existing representations, rather than a progressive decrease in the dimensionality of the control space.

Note that our results differentiate between schema-facilitated learning and structure learning. While a schema and an associated control space can emerge early, structure learning proceeds thereafter and involves learning to use the control space efficiently. Recent work has demonstrated the re-use of schematic prefrontal representations in rodents learning a series of odor-response sequence problems (Zhou et al. 2020). However, the authors did not observe an acceleration in learning. We propose that this may be explained by the presence of schema-facilitated learning, but an absence of structure learning. 
Crucially, our results offer experimentally verifiable predictions. First, the sensorimotor mapping schema is encoded by low-dimensional neural representations which are shared across problems, and explain a majority of the variance in population activity. They encode shared task variables including the task's temporal structure and the available choices. Second, the reuse of these representations to learn new problems causes a speedup in learning; preventing this reuse with recently developed BMI interventions (Sadtler et al. 2014) should produce pronounced learning deficits. Third, population activity may undergo large changes between the beginning and end of problem learning. However, across problems, these changes are restricted to a low-dimensional subspace of the activity. Fourth, the number of trials to learn a problem decreases exponentially as a function of the number of previously learned problems. Taken together, our results shed insights into the neural substrate of a sensorimotor mapping schema, the reason for which its reuse markedly improves learning efficiency, and the neural mechanisms of structure learning that gives rise to learning-to-learn. In doing so, they elucidate the neural mechanisms of learning-to-learn and present novel techniques to analyze learningto-learn in RNNs.

Acknowledgements: We thank G.R. Yang, N.Y. Masse, U.P. Obilinovic, L.Y. Tian, D.V. Buonomano and J. Jaramillo for fruitful discussions; and Y. Liu, K. Berlemont, A. Battista and P. Theodoni for critical comments on the manuscript. This work was supported by the National Institute of Health U-19 program grant no. 5U19NS107609-03 and the Office of Naval Research grant no. N00014-17-1-2041. 


\section{References}

Abraham, W. C. 2008. "Metaplasticity: tuning synapses and networks for plasticity". Nature Reviews Neuroscience 9 (5): 387-387.

Anderson, R. C., R. J. Spiro, and M. C. Anderson. 1978. "Schemata as scaffolding for the representation of information in connected discourse". American Educational Research Journal 15 (3): 433-440.

Asaad, W. F., G. Rainer, and E. K. Miller. 1998. "Neural activity in the primate prefrontal cortex during associative learning". Neuron 21 (6): 1399-1407.

Bao, P., et al. 2020. "A map of object space in primate inferotemporal cortex". Nature: $1-6$.

Bartlett, F. C. 1932. Remembering: A study in experimental and social psychology. Cambridge University Press.

Behrens, T. E., et al. 2018. "What is a cognitive map? Organizing knowledge for flexible behavior". Neuron 100 (2): 490-509.

Bernardi, S., et al. 2020. "The geometry of abstraction in the hippocampus and prefrontal cortex". Cell 183 (4): 954-967.

Bittner, K. C., et al. 2017. "Behavioral time scale synaptic plasticity underlies CA1 place fields". Science 357 (6355): 1033-1036.

Braun, D. A., C. Mehring, and D. M. Wolpert. 2010. "Structure learning in action". Behavioural Brain Research 206 (2): 157-165.

Brodt, S., et al. 2018. "Fast track to the neocortex: A memory engram in the posterior parietal cortex". Science 362 (6418): 1045-1048.

Bussey, T. J., S. P. Wise, and E. A. Murray. 2002. "Interaction of ventral and orbital prefrontal cortex with inferotemporal cortex in conditional visuomotor learning". $B e$ havioral Neuroscience 116 (4).

Chi, M. T., R. Glaser, and E. Rees. 1981. Expertise in problem solving. Tech. rep. Pittsburgh Univ PA Learning Research and Development Center. 
Cromer, J. A., M. Machon, and E. K. Miller. 2011. "Rapid association learning in the primate prefrontal cortex in the absence of behavioral reversals". Journal of Cognitive Neuroscience 23 (7): 1823-1828.

Doya, K. 2002. "Metalearning and neuromodulation". Neural Networks 15 (4-6): 495506.

Dubreuil, A. M., et al. 2021. "The role of population structure in computations through neural dynamics". bioRxiv: 2020-07.

Eacott, M., and D. Gaffan. 1992. "Inferotemporal-frontal disconnection: The uncinate fascicle and visual associative learning in monkeys". European Journal of Neuroscience 4 (12): 1320-1332.

Finn, C., P. Abbeel, and S. Levine. 2017. "Model-agnostic meta-learning for fast adaptation of deep networks". In International Conference on Machine Learning, 1126-1135. PMLR.

Fusi, S., et al. 2007. "A neural circuit model of flexible sensorimotor mapping: Learning and forgetting on multiple timescales". Neuron 54 (2): 319-333.

Gilboa, A., and H. Marlatte. 2017. "Neurobiology of schemas and schema-mediated memory". Trends in Cognitive Sciences 21 (8): 618-631.

Harlow, H. F. 1949. "The formation of learning sets". Psychological Review 56 (1).

Hebscher, M., et al. 2019. "Rapid cortical plasticity supports long-term memory formation". Trends in Cognitive Sciences 23 (12): 989-1002.

Kaufman, M. T., et al. 2014. "Cortical activity in the null space: permitting preparation without movement". Nature neuroscience 17 (3): 440-448.

Kaufman, M. T., et al. 2016. "The largest response component in the motor cortex reflects movement timing but not movement type". Eneuro 3 (4).

Kobak, D., et al. 2016. "Demixed principal component analysis of neural population data". eLife 5 .

Lewis, P. A., and S. J. Durrant. 2011. "Overlapping memory replay during sleep builds cognitive schemata". Trends in Cognitive Sciences 15 (8): 343-351. 
McKenzie, S., et al. 2014. "Hippocampal representation of related and opposing memories develop within distinct, hierarchically organized neural schemas". Neuron 83 (1): $202-215$.

Passingham, R. 1995. The frontal lobes and voluntary action. Oxford University Press, New York.

Petrides, M. 1985. "Deficits on conditional associative-learning tasks after frontal-and temporal-lobe lesions in man". Neuropsychologia 23 (5).

Peysakhovich, B., E. A. Buffalo, and D. J. Freedman. Unpublished.

Piaget, J. 1926. The language and thought of the child. Harcourt Brace.

Preston, A. R., and H. Eichenbaum. 2013. "Interplay of hippocampus and prefrontal cortex in memory". Current Biology 23 (17): R764-R773.

Rumelhart, D. E. 1980. "Schemata: The building blocks". Theoretical issues in reading comprehension: Perspectives from cognitive psychology, linguistics, artificial intelligence and education: 33-58.

Rumelhart, D. E., and D. A. Norman. 1976. Accretion, tuning and restructuring: Three modes of learning. Tech. rep. Univ. California San Diego Center For Human Information Processing.

Sadtler, P. T., et al. 2014. "Neural constraints on learning". Nature 512 (7515): 423-426.

Sehgal, M., et al. 2013. "Learning to learn-intrinsic plasticity as a metaplasticity mechanism for memory formation". Neurobiology of Learning and Memory 105:186-199.

Steele, R. J., and R. G. Morris. 1999. "Delay-dependent impairment of a matchingto-place task with chronic and intrahippocampal infusion of the NMDA-antagonist D-AP5". Hippocampus 9 (2): 118-136.

Stringer, C., et al. 2019. "Spontaneous behaviors drive multidimensional, brainwide activity". Science 364 (6437).

Strogatz, S. H. 2016. Nonlinear dynamics and chaos: With applications to physics, biology, chemistry and engineering. Second edition. Taylor \& Francis Group, Oxford, Britain. 
Sussillo, D., and O. Barak. 2013. "Opening the black box: low-dimensional dynamics in high-dimensional recurrent neural networks". Neural Computation 25 (3): 626-649.

Tenenbaum, J. B., et al. 2011. "How to grow a mind: Statistics, structure, and abstraction". Science 331 (6022): 1279-1285.

Thorndyke, P. W., and B. Hayes-Roth. 1979. "The use of schemata in the acquisition and transfer of knowledge". Cognitive Psychology 11 (1): 82-106.

Tse, D., et al. 2007. "Schemas and memory consolidation". Science 316 (5821): 76-82.

Tse, D., et al. 2011. "Schema-dependent gene activation and memory encoding in neocortex". Science 333 (6044): 891-895.

Van Kesteren, M. T. R., et al. 2012. "How schema and novelty augment memory formation". Trends in Neurosciences 35 (4): 211-219.

Van Kesteren, M. T., et al. 2013. "Differential roles for medial prefrontal and medial temporal cortices in schema-dependent encoding: From congruent to incongruent". Neuropsychologia 51 (12): 2352-2359.

Vyas, S., et al. 2020. "Computation through neural population dynamics". Annual Review of Neuroscience 43:249-275.

Wang, J. X., et al. 2018. "Prefrontal cortex as a meta-reinforcement learning system". Nature Neuroscience 21 (6): 860-868.

Wang, S.-H., and R. G. Morris. 2010. "Hippocampal-neocortical interactions in memory formation, consolidation, and reconsolidation". Annual Review of Psychology 61 (1): $49-79$.

Wang, S.-H., D. Tse, and R. G. Morris. 2012. "Anterior cingulate cortex in schema assimilation and expression". Learning \& Memory 19 (8): 315-318.

Zhou, J., et al. 2020. "Evolving schema representations in orbitofrontal ensembles during learning". Nature: 1-6. 
807

\section{Methods}

\section{Recurrent Neural Network Model (Fig. 1)}

The RNN model comprises a fully-connected population of $N$ firing rate units with firing rates $\boldsymbol{r}$, receiving inputs from $N_{i n}$ input units with firing rates $\boldsymbol{u}$. Firing rates of the network units follow the dynamical equation

$$
\begin{aligned}
\tau \dot{\boldsymbol{r}} & =-\boldsymbol{r}+f\left(W_{i n} \boldsymbol{u}+W_{r e c} \boldsymbol{r}+\boldsymbol{b}_{\boldsymbol{r e c}}+\boldsymbol{\zeta}\right) \\
\tau_{\zeta} \dot{\boldsymbol{\zeta}} & =-\boldsymbol{\zeta}+\sqrt{2 \tau_{\zeta} \sigma_{r e c}^{2}} \boldsymbol{\xi}
\end{aligned}
$$

which expresses the leaky and non-linear integration of input $\left(W_{i n} \boldsymbol{u}\right)$ and recurrent $\left(W_{\text {rec }} \boldsymbol{r}\right)$ currents. $W_{\text {in }}\left(W_{\text {rec }}\right)$ is an $N \times N_{\text {in }}(N \times N)$ matrix of input (recurrent) connection weights, and $\tau=100 \mathrm{~ms}$ is the integration time-constant that characterizes the slow decay of NMDA receptor-mediated synaptic currents (Wang 2002). The f-I curve is modeled by a smooth rectification function

$$
f(x)=\log \left(1+e^{x}\right)
$$

The bias term $\boldsymbol{b}_{\boldsymbol{r e c}}$ admits per-unit firing thresholds. Intrinsic background noise current is modeled by an Ornstein-Uhlenbeck process $\boldsymbol{\zeta}$ with time constant $\tau_{\zeta}$ and variance $\sigma_{\text {rec }}$, where $\boldsymbol{\xi}$ represents the underlying independent white-noise process with zero mean and unit variance.

Output responses are readout from the activity of the RNN units by $N_{\text {out }}$ output units, $\boldsymbol{y}$, whose activity is given by

$$
\boldsymbol{y}=g\left(W_{\text {out }} \boldsymbol{r}+\boldsymbol{b}_{\text {out }}\right)
$$

Here, $W_{\text {out }}$ is a $N_{\text {out }} \times N$ output weight matrix, $\boldsymbol{b}_{\text {out }}$ is the bias of the output units, and $g\left(x_{i}\right)=\exp \left(x_{i}\right) / \sum_{j=1}^{N_{\text {out }}} \exp \left(x_{j}\right)$ is the softmax or normalized-exponential function which 
produces output unit activity that indicates the probability of generating each of the $N_{\text {out }}$ response choices.

The model is simulated by temporal discretization of equation (1) with Euler's method, as

$$
\begin{aligned}
\boldsymbol{r}_{\boldsymbol{t}} & =(1-\alpha) \boldsymbol{r}_{\boldsymbol{t}-\mathbf{1}}+\alpha f\left(W_{i n} \boldsymbol{u}_{\boldsymbol{t}}+W_{\text {rec }} \boldsymbol{r}_{\boldsymbol{t}-\mathbf{1}}+\boldsymbol{b}_{\boldsymbol{r e c}}+\boldsymbol{\zeta}_{\boldsymbol{t}}\right) \\
\boldsymbol{\zeta}_{\boldsymbol{t}} & =\left(1-\alpha_{\zeta}\right) \boldsymbol{\zeta}_{\boldsymbol{t}-\mathbf{1}}+\sqrt{2 \alpha_{\zeta} \sigma_{r e c}^{2}} \mathcal{N}(\mathbf{0}, I)
\end{aligned}
$$

where the time-discretization step size is $\Delta t, \alpha=\Delta t / \tau, \alpha_{\zeta}=\Delta t / \tau_{\zeta}$ and $\mathcal{N}(\mathbf{0}, I)$ is a random vector sampled from a gaussian distribution with zero mean and identity covariance $(I)$. In all figures, the network size $N=100, \Delta t=1 \mathrm{~ms}, \tau_{\zeta}=2 \mathrm{~ms}$ and $\sigma_{r e c}=0.05$. The magnitude of the network- and input-unit firing rates is measured as the $L^{2}$-norm of $\boldsymbol{r}_{\boldsymbol{t}}$ and $\boldsymbol{u}_{\boldsymbol{t}}$, respectively, and summarized by averaging over all time points in a trial.

\section{Task Structure (Fig. 1)}

We trained the network model on a series of delayed sensorimotor association problems, one at a time. In each problem, the network had to learn a one-to-one correspondence between a pair of sample stimuli and a pair of motor responses. Each problem therefore comprised two trial types, one per stimulus-response pair. Each trial was $2 \mathrm{~s}$ in duration $(T=2)$, and started with a $500 \mathrm{~ms}$ sample epoch, followed by a $1 \mathrm{~s}$ delay epoch, and ended with a $500 \mathrm{~ms}$ choice epoch. During the sample epoch, the network concurrently received inputs representing a fixation stimulus and one sample stimulus. During the delay epoch, it continued to receive only the fixation input. It received no inputs during the choice epoch. The model was required to respond by maintaining fixation during the sample and delay epochs, and choosing the appropriate motor response during the choice epoch. Therefore, the model contained three output units $\left(N_{\text {out }}=3\right)$, two to report response choices and one for fixation. This trial structure, including the available 
response choices, remained fixed across problems.

Sample stimuli were represented by ten-dimensional unit-length vectors $\left(L^{2}\right.$-norm $=1$ ). The two sample stimulus input representations in a problem were drawn from a random gaussian distribution with zero mean and identity covariance. They were then orthogonalized to avoid learning efficiency confounds stemming from the relative difficulty in learning to distinguish between more versus less correlated sample stimuli. The fixation input was a scalar with value $1 / \sqrt{N_{i n}-1}$ when it was on and zero when off. Therefore, there were a total of $\operatorname{Nin}=11$ input units. Learning-to-learn was robustly observed even in the absence of the orthogonalization step; however, the variance in learning efficiency was higher. Qualitatively similar learning-to-learn performance was also observed with 200-dimensional sample stimulus representations and $N=1000$.

Each problem was learned over a sequence of trials, psueudorandomly sampled from the two trial types, until the average error on fifty consecutive trials fell below a criterion value (see Network Training). The learning efficiency for a problem was measured by the number of trials required to achieve this criterion. After a problem was learned, the model was transitioned to the next problem, wherein it had to learn to associate a new pair of pseudorandomly selected sample stimuli to the two motor responses.

\section{Network Training (Fig. 1)}

A network was trained on a problem by updating its connection weights $\left(W_{i n}, W_{\text {rec }}\right.$ and $\left.W_{\text {out }}\right)$, biases $\left(\boldsymbol{b}_{\boldsymbol{r e c}}\right.$ and $\left.\boldsymbol{b}_{\text {out }}\right)$ and initial network state $\left(\boldsymbol{r}_{\mathbf{0}}\right)$, so that it could choose the desired response for each of the sample stimuli. These updates were generated by stochastic gradient descent - an optimization algorithm that incrementally updates a network's parameters at the end of each trial, based on the errors in the output unit responses during the trial. In contrast to standard RNN training practices wherein model parameters are adjusted based on the average error from a batch of several trials and learning efficiency is measured by the number of trial batches to reach criterion performance, our training procedure closely matched established animal training proto- 
cols and allowed learning efficiency to be measured by the number of trials to criterion performance. The backpropagation through time (BPTT) algorithm was used to resolve temporal contingencies while computing parameter updates. We additionally applied the ADAM optimizer (Kingma and Ba 2014) to enhance the efficacy of the updates. All networks were trained with a learning rate of $10^{-4}$, except in Supplementary Figure 1 where the learning rate was systematically varied. ADAM decay rates for the first and second moment estimates were set to 0.3 and 0.999 , respectively, and the moment estimates were reset at the beginning of each problem. The model implementation and parameter update computations were performed with Tensorflow (Abadi et al. 2015).

Prior to the first problem, a naive network's input weights in $W_{i n}$ were initialized with random values drawn from a gaussian distribution with zero mean and variance $1 / N_{i n}$; the recurrent weights in $W_{\text {rec }}$ were initialized with random values constrained by householder transformations such that the rows (and columns) of the initial recurrent weight matrix were orthogonal to each other and of unit length (Stewart 1980). Initializing the recurrent weights in this manner allows gradients to be backpropagated more effectively. All other network parameters were initialized to zero. Upon transition to a new problem, all parameters retained their values. At initialization and throughout learning, the sign and sparsity of the weights and biases were not constrained. The initial network state was always restricted to non-negative values.

Network training was performed in a supervised setting, wherein the parameters were adjusted to minimize an objective function, $\mathcal{L}$, that included the errors in the model's output responses:

$$
\mathcal{L}_{\text {err }}=\frac{1}{T-\left|D_{\text {mask }}\right|} \sum_{t \notin D_{\text {mask }}} \sum_{i=1}^{N_{\text {out }}}-\check{y}_{i, t} \log \left(y_{i, t}\right)
$$

The error at each time step, $t$, was given by the cross-entropy of the probability distribution over responses generated by the network, $\boldsymbol{y}_{\boldsymbol{t}}$, relative to pre-specified target responses, $\check{\boldsymbol{y}}_{\boldsymbol{t}}$. The total error for a trial, $\mathcal{L}_{\text {err }}$, was the mean of the per-timestep error taken over the trial duration $T$. This mean excluded a masking interval, $D_{\text {mask }}$, set to 
the first $100 \mathrm{~ms}$ of the choice epoch, which allowed for flexible reaction times. Networks were considered to have learned a problem when the average $\mathcal{L}_{\text {err }}$ over fifty consecutive trials of the problem fell below a criterion value of 0.005 .

The objective of the training procedure was to minimize the sum of this error and auxiliary regularization terms:

$$
\mathcal{L}=\mathcal{L}_{\text {err }}+\mathcal{L}_{\text {reg, } W_{\text {in }}}+\mathcal{L}_{\text {reg, }, W_{\text {out }}}+\mathcal{L}_{\text {reg }, W_{\text {rec }}}+\mathcal{L}_{\text {reg, rate }}
$$

The regularization terms included both weight and activity regularization to encourage solutions that generalized well (Krogh and Hertz 1992; Merity et al. 2017) and generated stable network dynamics. We imposed $L^{2}$ regularization on the input and output weights as follows:

$$
\mathcal{L}_{\text {reg, }, W_{i n}}=\frac{\beta_{W_{i n}}}{N_{i n} N} \sum_{i=1}^{N_{i n}} \sum_{j=1}^{N}\left(W_{i n}(j, i)\right)^{2}
$$

$$
\mathcal{L}_{\text {reg }, W_{\text {out }}}=\frac{\beta_{W_{\text {out }}}}{N_{\text {out }} N} \sum_{i=1}^{N} \sum_{j=1}^{N_{\text {out }}}\left(W_{\text {out }}(j, i)\right)^{2}
$$

We observed that networks with a similar $L^{2}$ regularization of the recurrent weights were sensitive to the value of meta-parameter $\beta_{W_{r e c}}$, particularly when the network size was large - small values of $\beta_{W_{\text {rec }}}$ produced unstable network dynamics during later problems, while large values hindered learning efficiency. The squared frobenius norm of the recurrent weight matrix, which constitutes such an $L^{2}$ regularization, is given by:

$$
\sum_{i=1}^{N} \sum_{j=1}^{N}\left(W_{r e c}(j, i)\right)^{2}=\sum_{i=1}^{N} \sigma_{i}^{2}
$$

where $\sigma_{i}$ is the $i^{\text {th }}$ singular value of the recurrent weight matrix $W_{\text {rec }}$.

An analysis of these singular values under conditions that led to unstable network dynamics revealed that their $L^{2}$-norm (i.e. the square root of the right-hand side of the equation above) remained roughly fixed over the course of learning several problems; However, their distribution changed considerably across problems - smaller singular values shrank, while larger singular values grew and ultimately resulted in unstable network responses to novel sample stimuli. We mitigated this by introducing an alternate form 
of recurrent weight regularization that penalized the magnitude of the first $k$ singular values of $W_{\text {rec }}$ :

$$
\mathcal{L}_{\text {reg }, W_{\text {rec }}}=\frac{\beta_{W_{\text {rec }}}}{N k} \sum_{i=1}^{k} \sigma_{i}^{2}
$$

Finally, we imposed a homeostatic firing rate regularization:

$$
\mathcal{L}_{\text {reg,rate }}=\beta_{r}\left|\frac{1}{N T} \sum_{t} \sum_{i=1}^{N} r_{i, t}^{2}-h\right|
$$

The meta-parameter $h$ was set to zero for the first problem, effectively imposing an $L^{2}$ regularization of the recurrent unit firing rates as the first problem was learned. To avoid unrestrained growth or reduction in the firing rates while learning subsequent problems, the homeostatic set-point $h$ was then set to the mean squared firing rates averaged over the last fifty trials of the first problem. All networks were trained with $\beta_{W_{i n}}=10^{-4}$, $\beta_{W_{\text {rec }}}=0.1, \beta_{W_{\text {out }}}=0.1, k=10$ and $\beta_{r}=5 \times 10^{-4}$, except in Supplementary Figure 1, where these hyper-parameters were systematically varied.

\section{Learning-to-learn Performance Characterization (Fig. 1)}

A network exhibits learning-to-learn if its learning efficiency improves as a function of the number of previously learned problems. We evaluated this by quantifying the relationship between the trials-to-criterion on a problem and the number of problems learned thus far, where a decreasing relationship indicates learning-to-learn. Specifically, we fit a decaying exponential function to the number of trials to criterion $l(p)$ on problem $p$, as a function of the number of learned problems $p-1$ :

$$
l(p)=s_{l} \exp \left(\frac{-(p-1)}{\tau_{l}}\right)+a_{l}
$$

Here, $a_{l}$ represents asymptotic learning efficiency, $\tau_{l}$ represents the time-constant to achieve this asymptote, and $s_{l}$ represents the improvement in learning efficiency between early and late problems. A large asymptote signifies poor learning-to-learn, while a large time-constant signifies slow learning-to-learn. The three parameters of the function 
were fit with the Levenberg-Marquardt algorithm implemented by the fit function of MATLAB's curve fitting toolbox. The learning efficiency on the first problem was excluded from this analysis.

\section{Subspace Decomposition (Fig. 2)}

We performed semi-supervised dimensionality reduction on the population activity, to determine how strongly and consistently the shared task structure is represented across problems. The procedure begins by compiling a tensor $R_{k, t, j, i}$ of activity patterns generated by the population of firing rate units $(k \in[1, N])$ over time $(t \in(0, T])$, for the two response types $\left(j \in\left\{\right.\right.$ response $_{1}$, response $\left.\left._{2}\right\}\right)$ across a group of fifty consecutively learned problems $(i \in[p+1, p+50])$. This assembles a tensor of one hundred population trajectories for the group, fifty for each response type. The semi-supervised dimensionality reduction extracts decision representations that are shared by the group as follows. Stimulus- and problem-specific representations for each response type are averaged out, or marginalized, across problems in the group:

$$
R_{k, t, j, .}=<R_{k, t, j, i}>_{i}
$$

Principal components analysis is performed on a concatenation of the resulting two trajectories in $R_{k, t, j, .}$. The loading vectors for the first $m$ principal components are collected into a $N \times m$ loading matrix $L_{D}$. These vectors define a basis for the decision subspace. Importantly, to ensure that the decision subspace fully captures shared decision representations, the marginalized trajectories are not de-meaned before performing principal components analysis. Here, we set $m$ to 4 , as the first 4 principal components collectively explained at least $98 \%$ of the variance in the marginalized trajectories, in all the networks we analyzed.

Next, an $N \times N$ projection matrix $P(Q)$ that projects population activity into the decision subspace (stimulus subspace), is defined as:

$$
P=L_{D} L_{D}^{T}
$$


974

$$
Q=I-P
$$

where $I$ is the identity matrix. The decision components of the learned trajectories for problem $p+x(x \in[1,50])$ are identified as:

$$
R_{k 1, t, j, i=p+x}^{d}=\sum_{k 2=1}^{N} P(k 1, k 2) R_{k 2, t, j, i=p+x}
$$

and their stimulus components as:

$$
R_{k 1, t, j, i=p+x}^{s}=\sum_{k 2=1}^{N} Q(k 1, k 2) R_{k 2, t, j, i=p+x}
$$

The decision components are further decomposed into mean $\left(R_{k, t, j, i=p+x}^{d m}\right)$ and residual $\left(R_{k, t, j, i=p+x}^{d r}\right)$ decision components, as:

$$
R_{k, t, ., i=p+x}^{d m}=<R_{k, t, j, i=p+x}^{d}>_{j}
$$

$$
R_{k, t, j, i=p+x}^{d r}=R_{k, t, j, i=p+x}^{d}-R_{k, t,, i=p+x}^{d m}
$$

The net current from these components $R_{k, t, j, i=p+x}^{\nu}(\nu=\in\{s, d m, d r\})$ to an output unit $o$ was computed as $\sum_{k=1}^{N} W_{\text {out }}^{p+x}(o, k) R_{k, t, j, i=p+x}^{\nu}$ where $W_{\text {out }}^{p+x}$ is the output weight matrix learned in problem $p+x$. The dimensionality of any set of vectors (e.g. population activity in the stimulus subspace) was approximated by its participation ratio (Gao et al. 2017), computed as $\frac{\left(\sum_{i} \lambda_{i}\right)^{2}}{\sum_{i} \lambda_{i}^{2}}$, where $\lambda_{i}$ is the $i^{\text {th }}$ eigenvalue of the covariance matrix of the vectors.

\section{Manifold Perturbations (Fig. 3)}

To assess whether the reuse of the decision representations improves learning efficiency, networks were trained on their second problem while constraining them in a manner that required the formation of new decision representations. The learning efficiency of such networks was compared to controls that were allowed to reuse existing decision representations while learning their second problem. 
A naive network was first trained on 50 problems and the corresponding populations trajectories were used to identify its decision and stimulus subspaces. The network's parameters, including its output weights, were reset to their values at the end of the first problem. Then, its output weights were perturbed, and the network was trained on a new problem, i.e. a second problem with respect to its parameters, while barring the training procedure from changing its output weights. This procedure was repeated fifty times for each network, resetting its parameters, applying an independently chosen random perturbation to its output weights, freezing the output weights, and training the network on a new sample stimulus pair each time. The output weights were subjected to one of three forms of perturbation. In the frozen readout condition, the output weights were unperturbed after the parameter reset. In $\mathrm{D} \rightarrow \mathrm{S}$ manifold perturbations, following the parameter reset, the output weights were perturbed to replace the overlap between the network's readout and decision subspaces with a corresponding overlap between its readout and stimulus subspaces:

$$
W_{\text {out }, D \rightarrow S}=W_{\text {out }}-\sum_{i=1}^{4} W_{\text {out }} \boldsymbol{l}_{\boldsymbol{i}}^{\boldsymbol{D}} \boldsymbol{l}_{\boldsymbol{i}}^{\boldsymbol{D}^{T}}+\sum_{i=1}^{4} W_{\text {out }} \boldsymbol{l}_{\boldsymbol{i}}^{\boldsymbol{D}} \boldsymbol{l}_{\boldsymbol{\sigma}(\boldsymbol{i})}^{\boldsymbol{S}^{T}}
$$

where $W_{\text {out }, D \rightarrow S}$ is the perturbed output weight matrix, $\boldsymbol{l}_{\boldsymbol{i}}^{\boldsymbol{D}}\left(\boldsymbol{l}_{\boldsymbol{i}}^{\boldsymbol{S}}\right)$ is the $i^{\text {th }}$ principal component loading vector of the decision (stimulus) subspace, and $\sigma()$ represents a random shuffle or permutation of the stimulus subspace principal component loading vectors. In $\mathrm{S} \rightarrow \mathrm{S}$ manifold perturbations, following the parameter reset, the output weights were perturbed to permute the overlap between the readout and stimulus subspaces:

$$
W_{\text {out }, S \rightarrow S}=W_{\text {out }}-\sum_{i=1}^{4} W_{\text {out }} \boldsymbol{l}_{\boldsymbol{i}}^{\boldsymbol{S}} \boldsymbol{l}_{\boldsymbol{i}}^{\boldsymbol{S}^{T}}+\sum_{i=1}^{4} W_{\text {out }} \boldsymbol{l}_{\boldsymbol{i}}^{\boldsymbol{S}} \boldsymbol{l}_{\boldsymbol{\sigma}(\boldsymbol{i})}^{\boldsymbol{S}^{T}}
$$




\section{Relationship between pre-learning and learned trajectories based on weight- and state-driven vector field changes (Figs. 4- 5)}

Over the course of learning problem $p$, the model's parameters change from their values at the beginning of the problem, i.e. their pre-learning values $\left(W_{i n}^{p-1}, W_{r e c}^{p-1}, \boldsymbol{b}_{r e c}^{p-1}, W_{\text {out }}^{p-1}\right.$, $\boldsymbol{b}_{\text {out }}^{\boldsymbol{p}-\mathbf{1}}$ and $\left.\boldsymbol{r}_{\mathbf{0}}^{\boldsymbol{p}-\mathbf{1}}\right)$, to their values at the end of the problem, i.e. their learned values $\left(W_{i n}^{p}\right.$, $W_{\text {rec }}^{p}, \boldsymbol{b}_{\boldsymbol{r e c}}^{\boldsymbol{p}}, W_{\text {out }}^{p}, \boldsymbol{b}_{\text {out }}^{\boldsymbol{p}}$ and $\boldsymbol{r}_{\mathbf{0}}^{\boldsymbol{p}}$ ). The difference between the learned and pre-learning values of the parameters quantify their change due to learning problem $p\left(\Delta W_{i n}^{p}, \Delta W_{r e c}^{p}\right.$, $\Delta \boldsymbol{b}_{\boldsymbol{r e c}}^{\boldsymbol{p}}, \Delta W_{\text {out }}^{p}, \Delta \boldsymbol{b}_{\text {out }}^{\boldsymbol{p}}$ and $\left.\Delta \boldsymbol{r}_{\mathbf{0}}^{\boldsymbol{p}}\right)$, and are collectively referred to as $\Delta W^{p}$.

Due to these parameter changes, the population activity in response to inputs $\boldsymbol{u}_{\boldsymbol{t}}^{p}$ is altered from its pre-learning levels, $\boldsymbol{r}_{\boldsymbol{t} \in[\mathbf{0}, \boldsymbol{T}]}^{p}$, to its learned ones, $\boldsymbol{r}_{\boldsymbol{t} \in[\mathbf{0 , \boldsymbol { T } ]}}^{\boldsymbol{p}}$ (Fig. $4 \mathrm{~b}$, left). We derive an expression for this change in population activity, $\boldsymbol{z}_{\boldsymbol{t} \in[\mathbf{0 , T}]}^{\boldsymbol{T}}$, in terms of the parameter changes. Based on the time-discretized model equation (2), we have:

$$
\begin{aligned}
& z_{t}^{p}=r_{t}^{p}-r_{t}^{\prime p} \\
& =\left[(1-\alpha) \boldsymbol{r}_{t-\mathbf{1}}^{\boldsymbol{p}}+\alpha f\left(W_{i n}^{p} \boldsymbol{u}_{\boldsymbol{t}}^{p}+W_{r e c}^{p} \boldsymbol{r}_{t-\mathbf{1}}^{p}+\boldsymbol{b}_{\boldsymbol{r e c}}^{\boldsymbol{p}}\right)\right]- \\
& {\left[(1-\alpha) \boldsymbol{r}_{\boldsymbol{t}-\mathbf{1}}^{\prime p}+\alpha f\left(W_{i n}^{p-1} \boldsymbol{u}_{\boldsymbol{t}}^{p}+W_{r e c}^{p-1} \boldsymbol{r}_{\boldsymbol{t}-\mathbf{1}}^{\prime p}+\boldsymbol{b}_{\boldsymbol{r e c}}^{\boldsymbol{p}-\mathbf{1}}\right)\right]} \\
& =\left[\boldsymbol{r}_{t-1}^{p}-\boldsymbol{r}_{t-1}^{\prime p}\right]+\alpha\left[-\boldsymbol{r}_{t-1}^{p}+f\left(W_{i n}^{p} \boldsymbol{u}_{t}^{p}+W_{r e c}^{p} \boldsymbol{r}_{t-1}^{p}+\boldsymbol{b}_{\boldsymbol{r e c}}^{\boldsymbol{p}}\right)\right]- \\
& \alpha\left[-\boldsymbol{r}_{\boldsymbol{t}-\mathbf{1}}^{\prime p}+f\left(W_{i n}^{p-1} \boldsymbol{u}_{\boldsymbol{t}}^{p}+W_{r e c}^{p-1} \boldsymbol{r}_{\boldsymbol{t}-\mathbf{1}}^{\prime p}+\boldsymbol{b}_{\boldsymbol{r e c}}^{\boldsymbol{p - 1}}\right)\right] \\
& =\left[\boldsymbol{r}_{t-1}^{p}-\boldsymbol{r}_{t-1}^{\prime p}\right]+\alpha\left[-\boldsymbol{r}_{t-1}^{p}+f\left(W_{i n}^{p} \boldsymbol{u}_{t}^{p}+W_{r e c}^{p} \boldsymbol{r}_{t-1}^{p}+\boldsymbol{b}_{\boldsymbol{r e c}}^{\boldsymbol{p}}\right)\right]- \\
& \alpha\left[-\boldsymbol{r}_{\boldsymbol{t}-\mathbf{1}}^{\prime p}+f\left(W_{i n}^{p-1} \boldsymbol{u}_{\boldsymbol{t}}^{\boldsymbol{p}}+W_{\text {rec }}^{p-1} \boldsymbol{r}_{\boldsymbol{t}-\mathbf{1}}^{\prime p}+\boldsymbol{b}_{\boldsymbol{r e c}}^{\boldsymbol{p}-\mathbf{1}}\right)\right]+ \\
& \alpha\left[-\boldsymbol{r}_{\boldsymbol{t}-\mathbf{1}}^{\boldsymbol{p}}+f\left(W_{\text {in }}^{p-1} \boldsymbol{u}_{\boldsymbol{t}}^{\boldsymbol{p}}+W_{\text {rec }}^{p-1} \boldsymbol{r}_{\boldsymbol{t}-\mathbf{1}}^{\boldsymbol{p}}+\boldsymbol{b}_{\boldsymbol{r e c}}^{\boldsymbol{p}-\mathbf{1}}\right)\right]- \\
& \alpha\left[-\boldsymbol{r}_{\boldsymbol{t}-\mathbf{1}}^{p}+f\left(W_{i n}^{p-1} \boldsymbol{u}_{\boldsymbol{t}}^{p}+W_{r e c}^{p-1} \boldsymbol{r}_{\boldsymbol{t}-\mathbf{1}}^{\boldsymbol{p}}+\boldsymbol{b}_{\boldsymbol{r e c}}^{\boldsymbol{p}-\mathbf{1}}\right)\right]
\end{aligned}
$$


Rearranging the terms, we have:

$$
\begin{aligned}
& \boldsymbol{z}_{t}^{p}=\boldsymbol{z}_{t-1}^{p}+ \\
& \alpha\left[\left\{-\boldsymbol{r}_{t-1}^{p}+f\left(W_{i n}^{p-1} \boldsymbol{u}_{t}^{p}+W_{r e c}^{p-1} \boldsymbol{r}_{t-1}^{p}+\boldsymbol{b}_{r e c}^{p-1}\right)\right\}-\right. \\
&\left.\quad\left\{-\boldsymbol{r}_{t-\mathbf{1}}^{\prime p}+f\left(W_{i n}^{p-1} \boldsymbol{u}_{t}^{p}+W_{r e c}^{p-1} \boldsymbol{r}_{t-1}^{\prime p}+\boldsymbol{b}_{r e c}^{p-1}\right)\right\}\right]+ \\
& \alpha\left[f\left(W_{i n}^{p} \boldsymbol{u}_{t}^{p}+W_{r e c}^{p} \boldsymbol{r}_{t-1}^{p}+\boldsymbol{b}_{r e c}^{p}\right)-f\left(W_{i n}^{p-1} \boldsymbol{u}_{t}^{p}+W_{r e c}^{p-1} \boldsymbol{r}_{t-1}^{p}+\boldsymbol{b}_{r e c}^{p-1}\right)\right]
\end{aligned}
$$

This expression shows that the change in population activity emerges from an accumulation of activity change increments, $\Delta \boldsymbol{z}_{t}^{p}$ (Fig. $4 \mathrm{~b}$, center):

$$
\Delta z_{t}^{p}=z_{t}^{p}-z_{t-1}^{p}
$$

These increments are composed of two terms:

$$
\Delta z_{t}^{p}=\Delta \text { Field } s_{s, t}^{p}+\Delta \text { Field } d_{w, t}^{p}
$$

The first term, $\Delta \boldsymbol{F i e l} \boldsymbol{d}_{\boldsymbol{s}, \boldsymbol{t}}^{p}$, expresses the difference in the pre-learning vector field at the positions in state space along the learned $\left(\boldsymbol{r}_{t-1}^{p}\right)$ and pre-learning $\left(\boldsymbol{r}_{t-1}^{\prime p}\right)$ trajectories (Fig. $1 \mathrm{~b}$, bottom right). It is therefore referred to as the state-driven vector field change (or SdVFC):

$$
\begin{array}{r}
\Delta \text { Field }_{s, t}^{p}=\alpha\left[\left\{-\boldsymbol{r}_{t-1}^{p}+f\left(W_{i n}^{p-1} \boldsymbol{u}_{t}^{p}+W_{\text {rec }}^{p-1} \boldsymbol{r}_{t-1}^{p}+\boldsymbol{b}_{\text {rec }}^{p-1}\right)\right\}-\right. \\
\left.\left\{-\boldsymbol{r}_{t-1}^{\prime p}+f\left(W_{i n}^{p-1} \boldsymbol{u}_{t}^{p}+W_{r e c}^{p-1} \boldsymbol{r}_{t-\mathbf{1}}^{\prime p}+\boldsymbol{b}_{\text {rec }}^{p-1}\right)\right\}\right]
\end{array}
$$

The second term, $\Delta \boldsymbol{F i e l} \boldsymbol{d}_{\boldsymbol{w}, \boldsymbol{t}}^{p}$, expresses the change in the vector field at population states along the learned trajectory due to the parameter changes (Fig. $4 \mathrm{~b}$, top right). It is therefore referred to as the weight-driven vector field change (or WdVFC):

$$
\Delta \boldsymbol{F i e l} \boldsymbol{d}_{\boldsymbol{w}, \boldsymbol{t}}^{p}=\alpha\left[f\left(W_{i n}^{p} \boldsymbol{u}_{t}^{p}+W_{r e c}^{p} \boldsymbol{r}_{t-\mathbf{1}}^{p}+\boldsymbol{b}_{r e c}^{p}\right)-f\left(W_{i n}^{p-1} \boldsymbol{u}_{t}^{p}+W_{r e c}^{p-1} \boldsymbol{r}_{t-\mathbf{1}}^{p}+\boldsymbol{b}_{r e c}^{p-1}\right)\right]
$$


The WdVFC stems from the change in the net afferent currents to the population, $\Delta$ Current $_{w, t}^{p}$, due to the parameter changes (Fig. 4a):

$$
\begin{aligned}
& \Delta \boldsymbol{F i e l d} \boldsymbol{w}, t_{\boldsymbol{p}}^{\boldsymbol{p}}=\alpha\left[f\left(W_{i n}^{p} \boldsymbol{u}_{\boldsymbol{t}}^{p}+W_{\text {rec }}^{p} \boldsymbol{r}_{\boldsymbol{t}-\mathbf{1}}^{p}+\boldsymbol{b}_{\boldsymbol{r e c}}^{\boldsymbol{p}}\right)-f\left(W_{i n}^{p-1} \boldsymbol{u}_{\boldsymbol{t}}^{\boldsymbol{p}}+W_{r e c}^{p-1} \boldsymbol{r}_{\boldsymbol{t}-\mathbf{1}}^{p}+\boldsymbol{b}_{\boldsymbol{r e c}}^{\boldsymbol{p}-\mathbf{1}}\right)\right] \\
& =\alpha\left[f\left(\left(W_{i n}^{p-1}+\Delta W_{i n}^{p}\right) \boldsymbol{u}_{t}^{p}+\left(W_{r e c}^{p-1}+\Delta W_{r e c}^{p}\right) \boldsymbol{r}_{\boldsymbol{t}-\mathbf{1}}^{p}+\left(\boldsymbol{b}_{\boldsymbol{r e c}}^{p-1}+\Delta \boldsymbol{b}_{\text {rec }}^{p}\right)\right)-\right. \\
& \left.f\left(W_{i n}^{p-1} \boldsymbol{u}_{\boldsymbol{t}}^{p}+W_{r e c}^{p-1} \boldsymbol{r}_{\boldsymbol{t}-\mathbf{1}}^{\boldsymbol{p}}+\boldsymbol{b}_{\boldsymbol{r e c}}^{\boldsymbol{p - 1}}\right)\right] \\
& =\alpha\left[f\left(W_{i n}^{p-1} \boldsymbol{u}_{t}^{p}+W_{r e c}^{p-1} \boldsymbol{r}_{t-1}^{p}+\boldsymbol{b}_{r e c}^{p-1}+\Delta \text { Current } \boldsymbol{w}, \boldsymbol{t}^{p}\right)-\right. \\
& \left.f\left(W_{i n}^{p-1} \boldsymbol{u}_{t}^{p}+W_{r e c}^{p-1} \boldsymbol{r}_{t-1}^{p}+\boldsymbol{b}_{r e c}^{p-1}\right)\right]
\end{aligned}
$$

where $\Delta$ Current $\boldsymbol{u}_{\boldsymbol{w}, \boldsymbol{t}}^{p}$ is determined by $\Delta W_{i n}^{p}, \Delta W_{r e c}^{p}, \Delta \boldsymbol{b}_{\boldsymbol{r e c}}^{\boldsymbol{p}}$, as:

$$
\Delta \text { Current } \boldsymbol{w}, t^{p}=\Delta W_{i n}^{p} \boldsymbol{u}_{t}^{p}+\Delta W_{r e c}^{p} \boldsymbol{r}_{t-1}^{p}+\Delta \boldsymbol{b}_{r e c}^{p}
$$

The change in initial population state is defined as $\Delta \boldsymbol{z}_{\mathbf{0}}^{\boldsymbol{p}}=\Delta \boldsymbol{r}_{\mathbf{0}}^{\boldsymbol{p}}=\boldsymbol{r}_{\mathbf{0}}^{\boldsymbol{p}}-\boldsymbol{r}_{\mathbf{0}}^{\boldsymbol{p}-\mathbf{1}}$. We omit the contribution of this change from our analyses, as it consistently showed a negligible effect on the evolution of the learned trajectory and the activity changes, across all problems and networks tested.

The contribution of the two vector field change terms to the activity change increment, $\Delta \boldsymbol{z}_{t}^{\boldsymbol{p}}$, was measured by their magnitude along, or in the direction of, $\Delta \boldsymbol{z}_{\boldsymbol{t}}^{\boldsymbol{p}}$ (Fig. $4 \mathrm{~b}$, right). This was computed by vector projection, as:

$$
\mid \Delta \text { Field }\left._{\mu, t}^{p}\right|_{\Delta z_{\|}^{p}}=\Delta \boldsymbol{F i e l d} \boldsymbol{\mu}, t^{p} \cdot \widehat{\Delta \boldsymbol{z}_{t}^{p}}
$$

where $\mu \in\{w, s\}, \cdot$ represents the dot product operator, and $\widehat{\Delta \boldsymbol{z}_{t}^{p}}$ is the unit vector in the direction of $\Delta z_{t}^{p}\left(\widehat{\Delta z_{t}^{p}}=\frac{\Delta z_{t}^{p}}{\left\|\Delta z_{t}^{p}\right\|_{2}}\right)$. Therefore, the vector field change along $\Delta z_{t}^{p}$ is 
given by:

$$
\Delta \text { Field }{ }_{\mu, t}^{p} z_{\|}^{p}=\mid \Delta \text { Field }\left.d_{\mu, t}^{p}\right|_{\Delta z_{\|}^{p}} \widehat{\Delta z_{t}^{p}}
$$

The remainder of each vector field change term represents its components orthogonal to $\Delta z_{t}^{p}$ (Fig. 4h, right):

$$
\Delta \text { Field }_{\mu, t_{\Delta z_{\perp}^{p}}^{p}}=\Delta \text { Field }_{\mu, t}^{p}-\Delta \text { Field }_{\mu, t_{\Delta z_{\|}^{p}}^{p}}
$$

The magnitude of change in the input and recurrent connection weights was measured by their frobenius norm, $\left\|W^{p}-W^{p-1}\right\|_{F}=\sqrt{\sum_{i, j}\left(W^{p}(i, j)-W^{p-1}(i, j)\right)^{2}}$. We measured the individual contributions of changes in the input weights $\left(\Delta W_{i n}^{p}\right)$, recurrent weights $\left(\Delta W_{r e c}^{p}\right)$ and network unit biases $\left(\Delta b_{r e c}^{p}\right)$ to the weight-driven vector field change. Note that, the postsynaptic current changes can be linearly decomposed based on the contributions of these parameter changes: they are given by the 3 terms on the right-hand side of equation (9), which we denote as $\Delta$ Current $_{\mu, t}^{p}$ to signify postsynaptic current changes due to changes in the parameter $\mu\left(\mu \in\left\{W_{i n}, W_{\text {rec }}, \boldsymbol{b}_{r e c}\right\}\right)$. In contrast, the vector field change is a non-linear function of these parameter changes. Therefore, we formulated non-linear approximations of their contributions, which we 
denote as $\Delta \boldsymbol{F i e l d} \boldsymbol{\mu}, \boldsymbol{t}$. Such an approximation must solely depend on changes in the parameter $\mu$, and the approximations must collectively satisfy the following with an acceptably small approximation error:

$$
\Delta \text { Field }_{w, t}^{p} \approx \Delta \text { Field }_{W_{i n}, t}^{p}+\Delta \text { Field }_{W_{r e c}, t}^{p}+\Delta \text { Field }_{b_{r e c}, t}^{p}
$$

$\Delta \boldsymbol{F i e l d} \boldsymbol{d}_{\boldsymbol{\mu}, \boldsymbol{t}}^{\boldsymbol{p}}$ is a vector, whose elements $\Delta F_{\mu, t}^{p}(i)$, represent the approximate change in the firing rate of network unit $i$, at time $t$ along the learned trajectory for problem $p$, due to the changes in parameter $\mu$. From equations (8) and (9), we observe that it is related to the net postsynaptic current at unit $i$ due to the pre-learning parameter values, $C_{t}^{\prime p}(i)$, and the net change in the postsynaptic current at unit $i, \Delta C_{t}^{p}(i)$. These net currents are given by the summations $C_{t}^{\prime p}(i)=\sum_{j} C_{t}^{\prime p}(i, j)$ and $\Delta C_{t}^{p}(i)=\sum_{j} \Delta C_{t}^{p}(i, j)$, where $j$ denotes an individual connection/bias that contributes to the net current into unit $i$. These include its individual afferent input and recurrent connections weights and its bias. We use the notation $\Delta C_{\mu, t}^{p}(i)=\sum_{j \in \mu} \Delta C_{t}^{p}(i, j)$ to explicitly refer to the net contribution of changes in parameter $\mu$ to the postsynaptic current changes at unit $i$. We derive an expression for $\Delta F_{\mu, t}^{p}(i)$ via taylor-expansion of equation (7):

$$
\begin{aligned}
\Delta F_{t}^{p}(i)=\alpha & \left.f\left(C_{t}^{\prime p}(i)+\Delta C_{t}^{p}(i)\right)-f\left(C_{t}^{\prime p}(i)\right)\right] \\
=\alpha\left[f\left(C_{t}^{\prime p}(i)\right)+\sum_{j} \nabla f_{C_{t}^{\prime p}(i)}(j) \Delta C_{t}^{p}(i, j)+\right. & \\
& \left.\quad \frac{1}{2} \sum_{j} \sum_{k} H_{C_{t}^{\prime p}(i)}(j, k) \Delta C_{t}^{p}(i, j) \Delta C_{t}^{p}(i, k)+H . O . T .-f\left(C_{t}^{\prime p}(i)\right)\right] \\
=\alpha & {\left[\sum _ { \mu } \left(\sum_{j \in \mu} \nabla f_{C_{\mu, t}^{\prime p}(i)}(j) \Delta C_{\mu, t}^{p}(i, j)+\right.\right.} \\
& \left.\frac{1}{2} \sum_{j \in \mu} \sum_{k \in \mu} H_{C_{\mu, t}^{\prime p}(i)}(j, k) \Delta C_{\mu, t}^{p}(i, j) \Delta C_{\mu, t}^{p}(i, k)+H . O . T \cdot \mu\right)+ \\
& + \text { N.L.I.T. }]
\end{aligned}
$$




$$
\begin{aligned}
& =\alpha\left[\sum _ { \mu } \left(f\left(C_{t}^{\prime p}(i)\right)+\sum_{j \in \mu} \nabla f_{C_{\mu, t}^{\prime p}(i)}(j) \Delta C_{\mu, t}^{p}(i, j)+\right.\right. \\
& \frac{1}{2} \sum_{j \in \mu} \sum_{k \in \mu} H_{C_{\mu, t}^{\prime p}(i)}(j, k) \Delta C_{\mu, t}^{p}(i, j) \Delta C_{\mu, t}^{p}(i, k)+ \\
& \left.\left.H . O . T \cdot \mu-f\left(C_{t}^{\prime p}(i)\right)\right)+ \text { N.L.I.T. }\right] \\
& =\alpha\left[\sum_{\mu}\left(f\left(C_{t}^{\prime p}(i)+\Delta C_{\mu, t}^{p}(i)\right)-f\left(C_{t}^{\prime p}(i)\right)\right)+\text { N.L.I.T. }\right] \\
& =\sum_{\mu} \Delta F_{\mu, t}^{p}(i)+\alpha[N . L . I . T .] \\
& \approx \sum_{\mu} \Delta F_{\mu, t}^{p}(i)
\end{aligned}
$$

where $\nabla f_{C_{t}^{\prime p}(i)}$ and $H_{C_{t}^{\prime p}(i)}$ correspond to the gradient and hessian of $f$ for unit $i$, when the magnitude of its net postsynaptic current is $C_{t}^{\prime p}(i)$. H.O.T. corresponds to the higherorder terms of the taylor expansion, H.O.T. ${ }_{\mu}$ corresponds to the higher-order terms that only involve changes to parameter $\mu$, and N.L.I.T. corresponds to non-linear interactions between the terms due to changes in $W_{i n}, W_{r e c}$ and $\boldsymbol{b}_{r e c}$. From the derivation above, we have:

$$
\Delta \text { Field }_{\boldsymbol{\mu}, t}^{p}=\alpha\left[f\left(\text { Current }_{t}^{\prime p}+\Delta \text { Current }_{\mu, t}^{p}\right)-f\left(\text { Current }_{t}^{\prime p}\right)\right]
$$

This equation expresses the unique contribution of changes in the parameter $\mu$ to the vector field change. Furthermore, the collective contribution of the changes in the three parameters satisfy equation (12), subject to an approximation error of $\alpha \boldsymbol{N}$.L.I.T. We calculated the magnitude ( $L^{2}$-norm) of this error; at each trial timestep and in each network tested, this error was found to be less than 1\% (average across problems). In supplementary figures 3 - 4, we forego presenting the contribution of the change in network unit biases $\left(\Delta \boldsymbol{b}_{r e c}^{p}\right)$, as it consistently showed a negligible effect on the changes in postsynaptic currents and the vector field in all problems and networks tested. 
For figure 6, this approach was extended to estimate the unique contribution of the changes in recurrent connection weights from the decision $\left(W_{\text {rec }}^{D \rightarrow S, D}\right)$ and stimulus $\left(W_{r e c}^{S \rightarrow S, D}\right)$ subspaces to the vector field change, where $W_{r e c}^{D \rightarrow S, D}=W_{r e c} P$ and $W_{r e c}^{S \rightarrow S, D}=$ $W_{\text {rec }} Q$. To do so, we extended the parameter set in the derivation above to $\mu \in$ $\left\{W_{i n}, W_{r e c}^{D \rightarrow S, D}, W_{r e c}^{S \rightarrow S, D}, \boldsymbol{b}_{\text {rec }}\right\}$. Also, the decision and stimulus components of the vector field change due to recurrent weight changes were calculated as $\Delta \boldsymbol{F i e l d _ { W _ { r e c } ^ { S , D \rightarrow D , t } } ^ { S }}=$

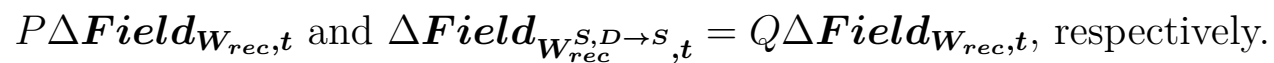

The clamping simulations to evaluate reciprocal interactions between stimulus and decision representations (Supplementary Fig. 6ic) were performed as follows. Starting from the initial population state $\left(\boldsymbol{r}_{\mathbf{0}}\right)$, the model was simulated for a single timestep with the learned parameter values as per equation (2). This advanced the population state to $\boldsymbol{r}_{\mathbf{1}}$. The stimulus (decision) representation was then reset to its pre-learning value $Q \boldsymbol{r}_{\mathbf{1}}^{\prime}\left(P \boldsymbol{r}_{\mathbf{1}}^{\prime}\right)$, and the model were simulated for another timestep. This process was repeated until the end of the trial. The Euclidean distance (or magnitude of deviation) between the decision (stimulus) representations observed during these simulations and the learned decision (stimulus) representations reflected the strength of the reciprocal interactions.

\section{Relationship between the accumulation of weight changes across problems and the progressive decrease in the weight-driven vec- tor field change (Fig. 7)}

We measured the contribution of the weight changes elicited while learning problem $p-k$ $\left(\Delta W^{p-k}\right.$, for $\left.1 \leq k \leq p-2\right)$ to the cumulative vector field change (CVFC) along the learned trajectory for problem $p\left(\Delta \boldsymbol{F} \boldsymbol{i e l d} \boldsymbol{d}_{\boldsymbol{w}, \boldsymbol{t}}^{\boldsymbol{p - k , p}}\right)$ as:

$$
\begin{aligned}
& \Delta \boldsymbol{F i e l d} \boldsymbol{d}_{\boldsymbol{w}, \boldsymbol{t}}^{\boldsymbol{p - k}, \boldsymbol{p}}=\alpha\left[f\left(W_{i n}^{p-k} \boldsymbol{u}_{\boldsymbol{t}}^{p}+W_{r e c}^{p-k} \boldsymbol{r}_{\boldsymbol{t}-\mathbf{1}}^{p}+\boldsymbol{b}_{\boldsymbol{r e c}}^{\boldsymbol{p - k}}\right)-\right. \\
& \left.f\left(W_{i n}^{p-k-1} \boldsymbol{u}_{\boldsymbol{t}}^{p}+W_{r e c}^{p-k-1} \boldsymbol{r}_{\boldsymbol{t}-\mathbf{1}}^{\boldsymbol{p}}+\boldsymbol{b}_{\boldsymbol{r e c}}^{\boldsymbol{p - k - 1}}\right)\right]
\end{aligned}
$$


Then, the cumulative vector field change due to the accumulation of weight changes across all the learned problems from $p-k$ to $p-1$ was given by:

$$
\begin{aligned}
& \sum_{j=1}^{k} \Delta \boldsymbol{F i e l d} \boldsymbol{w}, t^{p-j, p}=\alpha\left[f\left(W_{i n}^{p-1} \boldsymbol{u}_{t}^{p}+W_{r e c}^{p-1} \boldsymbol{r}_{t-1}^{p}+\boldsymbol{b}_{\boldsymbol{r e c}}^{p-1}\right)-\right. \\
& \left.f\left(W_{i n}^{p-k-1} \boldsymbol{u}_{\boldsymbol{t}}^{p}+W_{r e c}^{p-k-1} \boldsymbol{r}_{\boldsymbol{t}-\mathbf{1}}^{p}+\boldsymbol{b}_{\boldsymbol{r e c}}^{\boldsymbol{p - k - 1}}\right)\right]
\end{aligned}
$$

In figure 4, we present the magnitude of CVFC along the parallel $\left(\Delta \boldsymbol{z}_{\|}^{p}\right)$ and orthogonal $\left(\Delta \boldsymbol{z}_{\perp}^{\boldsymbol{p}}\right)$ components of the vector field change for problem $p$. These were computed via vector projection of the CVFC onto unit vectors in the direction of the vector field

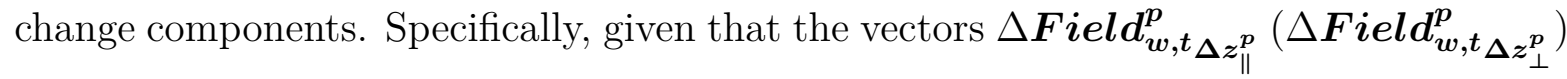
are nearly one-dimensional across trial time $t$ within problem $p$ (Fig. 5b), we applied principal components analysis to find a single basis (unit-norm) vector, $\Delta \widehat{\boldsymbol{F i e l d}}_{\boldsymbol{w}, \boldsymbol{e} \boldsymbol{\Delta} \boldsymbol{z}_{\|}^{p}}^{\boldsymbol{p}}$ $\left(\Delta \widehat{\boldsymbol{F i e l d}}_{\boldsymbol{w}, e_{\Delta z_{\perp}^{p}}^{p}}^{p}\right)$, that accurately represents their shared direction during each nonoverlapping $250 \mathrm{~ms}$ epoch, $e$, of the trial. The magnitude of the cumulative change along the parallel / orthogonal vector field change component was given by:

$$
\mid \sum_{j=1}^{k} \Delta \text { Field }\left._{w, t}^{p-j, p}\right|_{\Delta z_{\mu}^{p}}=\mid\left(\sum_{j=1}^{k} \Delta \text { Field }_{\boldsymbol{w}, t}^{p-j, p}\right) \cdot \widehat{\text { Field }}_{\boldsymbol{w}, e_{\Delta} z_{\mu}^{p}}^{p} \mid
$$

where $\mu \in\{\|, \perp\}$, and time $t$ lies within the interval of epoch $e$. The magnitudes of CVFC contribution by individual problems along the parallel / orthogonal vector field change component $\left(\mid \Delta\right.$ Field $\left.\left.d_{w, t}^{p-k, p}\right|_{\Delta z_{\mu}^{p}}\right)$ was computed similarly.

The signed CVFC and per-problem CVFC contributions in figure 7c were calculated as above, but without taking the absolute value on the right-hand-side.

The per-trial magnitude of the CVFC contribution by problem $p-k$ to problem $p$ was calculated as $\frac{\mid \Delta \text { Field }\left._{w, t}^{p-k, p}\right|_{\Delta z_{\mu}^{p}}}{l(p-k)}$, where $l(p-k)$ is the trials-to-criterion for problem $p-k$. The sum of the magnitudes of the CVFC contributions to problem $p$ was calculated as $\sum_{j=1}^{p-2} \mid \Delta$ Field $\left.d_{w, t}^{p-j, p}\right|_{\Delta z_{\mu}^{p}}$ 
The magnitude of net suppression of problem p's WdVFC along its parallel / orthogonal component is defined as the net suppression in the direction of the corresponding component due to net weight changes between the start of problems 2 and $p$. It was computed from the total vector field change along the learned trajectory for problem $p$ since the start of problem 2. Let $\Delta \boldsymbol{F i e l} \boldsymbol{d}_{\boldsymbol{w}, \boldsymbol{t}}^{\text {total, } \boldsymbol{p}}$ represent this total vector field change at time $t$ :

$$
\Delta \text { Field }_{w, t}^{\text {total }, p}=\sum_{j=1}^{p-2} \Delta \text { Field }_{w, t}^{p-j, p}+\Delta \text { Field }{ }_{w, t}^{p}
$$

Then the total change along the parallel / orthogonal vector field change component was given by:

$$
\Delta F_{w, t}^{t o t a l, p}{ }_{\Delta z_{\mu}^{p}}=\Delta \boldsymbol{F i e l d}_{\boldsymbol{w}, t}^{\text {total, } \boldsymbol{p}} \cdot \Delta \widehat{\boldsymbol{F i e l d}}_{\boldsymbol{w}, e_{\Delta} z_{\mu}^{p}}^{\boldsymbol{p}}
$$

We applied a sign correction to this quantity to ensure that its temporal mean is always positive. This allowed us to accurately calculate the net suppression. After sign correction, $\Delta F_{w, t}^{t o t a l, p}{ }_{\Delta z_{\mu}^{p}}$ becomes:

$$
\widetilde{\Delta F}_{w, t}^{t o t a l, p}{ }_{\Delta z_{\mu}^{p}}=\operatorname{sgn}\left(\Delta F_{w, .}^{\text {total, }}{ }_{\Delta z_{\mu}^{p}}^{p}\right) \Delta F_{w, t}^{\text {total }, p} \Delta z_{\mu}^{p}
$$

where $\Delta F_{w, .}^{\text {total, }}{ }_{\Delta z_{\mu}^{p}}$ represents the temporal mean of $\Delta F_{w, t}^{\text {total, } p}{ }_{\Delta z_{\mu}^{p}}$ over time $t$ within a trial, and $\operatorname{sgn}()$ represents the signum function. Similarly, the WdVFC for problem $p$ along its parallel / orthogonal components was given by:

$$
\Delta F_{w, t \Delta z_{\mu}^{p}}^{p}=\Delta \boldsymbol{F i e l d}_{\boldsymbol{w}, t}^{p} \cdot \Delta \widehat{\boldsymbol{F i e l d}}_{\boldsymbol{w}, e_{\Delta} z_{\mu}^{p}}^{p}
$$


Then, the magnitude of net suppression along the parallel / orthogonal vector field change component for problem $p$ was:

$$
\widetilde{\Delta F}_{w, t}^{\text {net }, p}{ }_{\Delta z_{\mu}^{p}}^{p}=\widetilde{\Delta F}_{w, t}^{\text {total,p}}{ }_{\Delta z_{\mu}^{p}}^{p}-\Delta F_{w, t \Delta z_{\mu}^{p}}^{p}
$$

The progression of this quantity over the learning-to-learn timecourse can be described in terms of the number of previously learned problems. We note that the temporal mean of the magnitude of the WdVFC along its parallel / orthogonal component $\left(\Delta F_{w, \Delta z_{\mu}^{p}}^{p}\right)$ decays exponentially from problem 2 onwards until an asymptotic value $b_{\mu}$ is converged upon (as in Fig. 6b). This decay may be expressed as:

$$
\left(\Delta F_{w, \cdot \Delta z_{\mu}^{p}}^{p}-b_{\mu}\right)=\left(\Delta F_{w, \cdot \Delta z_{\mu}^{2}}^{2}-b_{\mu}\right) r_{\mu}^{p-2}
$$

for an appropriate base $r_{\mu}<1$. Taking the temporal mean of equation (17) over trial time $t$, we have:

$$
\begin{aligned}
& \widetilde{\Delta F}_{w, \cdot \Delta z_{\mu}^{p}}^{n e t, p}=\widetilde{\Delta F}_{w, . \quad \Delta z_{\mu}^{p}}^{\text {total }, p}-\Delta F_{w, \cdot \Delta z_{\mu}^{p}}^{p} \\
& =\widetilde{\Delta F}_{w, .}^{t o t a l, p} \quad \Delta z_{\mu}^{p}-\left(\Delta F_{w, \cdot \Delta z_{\mu}^{p}}^{p}-b_{\mu}+b_{\mu}\right) \\
& =\widetilde{\Delta F}_{w, . \quad \Delta z_{\mu}^{p}}^{\text {total }, p}-\left(\Delta F_{w, \cdot \Delta z_{\mu}^{p}}^{p}-b_{\mu}\right)-b_{\mu} \\
& =\widetilde{\Delta F}_{w, .}^{\text {total }, p} \quad \Delta z_{\mu}^{p}-\left(\Delta F_{w, . \Delta z_{\mu}^{2}}^{2}-b_{\mu}\right) r_{\mu}^{p-2}-b_{\mu}
\end{aligned}
$$

Rearranging, we have:

$$
\widetilde{\Delta F}_{w, . \quad \Delta z_{\mu}^{p}}^{n e t, p}=\left(\widetilde{\Delta F}_{w, .}^{t o t a l, p} \quad \Delta z_{\mu}^{p}-b_{\mu}\right)-\left(\Delta F_{w, . \Delta z_{\mu}^{2}}^{2}-b_{\mu}\right) r_{\mu}^{p-2}
$$

This equation expresses the progression of the magnitude of net suppression over the learning-to-learn timecourse, and determines its shape as a function of the number of 
previously learned problems (Fig. $7 \mathrm{C})$. Note that when the first term $\left(\widetilde{\Delta F}_{w, . \quad \Delta z_{\mu}^{p}}^{\text {total,p }}-b_{\mu}\right)$ is roughly constant across learning-to-learn stages (as we found by measurement), the magnitude of net suppression is given by an inverted exponential function.

Finally, we determined the relative contributions of the cumulative input versus recurrent weight changes to the CVFC along the orthogonal vector field change component (Supplementary Fig. 7c). To do so, we calculated the CVFC for problem $p$ solely due to the accumulation of input weight changes elicited by previously learned problems as:

$\sum_{j=1}^{\boldsymbol{k}} \Delta \boldsymbol{F i e l} \boldsymbol{d}_{\boldsymbol{w}_{i n}, \boldsymbol{t}}^{\boldsymbol{p - j , p}}=\alpha\left[f\left(W_{i n}^{p} \boldsymbol{u}_{\boldsymbol{t}}^{\boldsymbol{p}}+W_{r e c}^{p} \boldsymbol{r}_{\boldsymbol{t}-\mathbf{1}}^{\boldsymbol{p}}+\boldsymbol{b}_{\boldsymbol{r e c}}^{\boldsymbol{p}}\right)-f\left(W_{i n}^{p-k-1} \boldsymbol{u}_{\boldsymbol{t}}^{\boldsymbol{p}}+W_{r e c}^{p} \boldsymbol{r}_{\boldsymbol{t}-\mathbf{1}}^{\boldsymbol{p}}+\boldsymbol{b}_{\boldsymbol{r e c}}^{\boldsymbol{p}}\right)\right]$

The CVFC solely to due to recurrent weight changes was calculated similarly. Both quantities were then projected onto the basis vector for the orthogonal vector field change components in problem $p$ (as in equation (16)), to compare their contributions along this component.

\section{References}

Abadi, M., et al. 2015. TensorFlow: Large-Scale Machine Learning on Heterogeneous Systems. Software available from tensorflow.org. https://www.tensorflow.org/.

Gao, P., et al. 2017. "A theory of multineuronal dimensionality, dynamics and measurement". BioRxiv.

Kingma, D. P., and J. Ba. 2014. "Adam: A method for stochastic optimization". arXiv preprint arXiv:1412.6980.

Krogh, A., and J. A. Hertz. 1992. "A simple weight decay can improve generalization". In Advances in Neural Information Processing Systems, 950-957.

Merity, S., B. McCann, and R. Socher. 2017. "Revisiting activation regularization for language RNNs". arXiv preprint arXiv:1708.01009. 
bioRxiv preprint doi: https://doi org/10.1101/2021.09.02.455707; this version posted September 2, 2021. The copyright holder for this preprint (which was not certified by peer review) is the author/funder, who has granted bioRxiv a license to display the preprint in perpetuity. It is made available under aCC-BY-NC 4.0 International license.

1182

1183

1184

Stewart, G. W. 1980. "The efficient generation of random orthogonal matrices with an application to condition estimators". SIAM Journal on Numerical Analysis 17 (3): 403-409.

Wang, X.-J. 2002. "Probabilistic decision making by slow reverberation in cortical circuits". Neuron 36 (5): 955-968. 


\section{Supplementary Notes}

\subsection{Recurrent plasticity elicits efficient learning, but is not nec- essary for it}

The reuse of the decision manifold to compose learned trajectories implies that a problem is learned by mapping each of its sample stimuli onto an appropriate decision representation within the decision manifold. The model may achieve this either by adjusting its input connection weights to appropriately remap the novel sensory inputs, or by adjusting its recurrent connection weights to alter how these inputs are recurrently integrated into the appropriate decision representations, or by some combination of the two. To assess the relative contributions of these two mechanisms, we compared: (i) the magnitude of change in the input and recurrent weights when problems are learned; (ii) the decrease in output accuracy when the input or recurrent weights changes are reversed; (iii) the learning-to-learn performance of networks with a pre-established representational manifold, that must exclusively rely on changes to either their input or recurrent weights to learn new problems.

We observed that the input weight changes were similar in magnitude to the recurrent weight changes (Supplementary Fig. 3a). Yet, reversing these relatively large input weight changes produced a negligible decrease in response accuracy. In contrast, reversing the recurrent weight changes decreased response accuracies to chance levels (Supplementary Fig. 3b). To address this discrepancy between the relative magnitude of the weight changes and their effect on output response accuracy, we approximated and compared the individual contributions of the input and recurrent weight changes to the WdVFC (equation (13), see Methods). Consistent with the latter result, we found that the WdVFC is primarily caused by recurrent weight changes (Supplementary Fig. 3c, left). Recall that the WdVFC is directly related to changes in the postsynaptic currents (Fig. 4a, equation (8)), which is a product of the connection weight changes and the firing rates of network and input units (equation (9)). Given that the input and recurrent weight changes are comparable in magnitude, we posited that the disproportionate 
contribution of the recurrent weight changes would be explained by a difference in the magnitudes of the network and input unit firing rates. A comparison of these firing rate magnitudes confirmed our hypothesis (Supplementary Fig. 4d, Input Act. Mag. = 1, $W_{\text {in }}$ gain $=1$, Baseline).

These results demonstrate that the model prefers solutions that rely on recurrent weight changes, and that these solutions make more efficient use of the weight changes to alter the vector field. But are these solutions preferred because they are more efficient to learn? In other words, do solutions that rely on input weight changes exhibit poor learning efficiency? To answer this question, we trained networks with pre-established decision and stimulus manifolds (i.e. networks trained on their first problem) on new problems, either with frozen input weights or with frozen recurrent weights. We then compared their asymptotic learning efficiency. Indeed, we found that networks with frozen recurrent weights exhibited substantially higher learning efficiency asymptotes than networks with frozen input weights (Supplementary Fig. 4c) - the model's preference for solutions that relied on recurrent weight changes was predicated on their superior learning efficiency. Moreover, networks with frozen recurrent weights required considerable changes to their input weights before they had learned a problem (Supplementary Fig. 4c, Input Act. Mag. $=1, W_{\text {in }}$ gain =1). This suggests that the model's learning efficiency on a problem is related to the magnitude of connection weights changes that are necessary to learn it. We further explore this relationship in the main text (Fig. 6).

In the networks explored thus far, learning is more efficient when it relies on recurrent weight changes. We sought to understand whether this is always true, i.e. are recurrent weight changes a necessary condition for efficient learning? Or, do network regimes exist wherein learning is equally efficient when driven by input weight changes? Networks that learn via input weight changes exhibit poorer learning efficiency due to deficits in their influence on the postsynaptic current changes and the WdVFC (Fig. 4d). Therefore, we reasoned that such networks may become efficient learners in regimes where this deficit is eliminated. To test this, we measured the asymptotic learning efficiency of networks with a tenfold increase in input unit firing rates, and with frozen recurrent weights. We expected that this increase would facilitate a stronger influence of input weight changes 
on postsynaptic current changes, thereby improving learning efficiency. Surprisingly, we found that these networks also under-performed in comparison to networks with plastic recurrent weights (Supplementary Fig. 4tb, Input Act. Mag. $=10, W_{\text {in }}$ gain =1). Consistent with the original networks (Input Act. Mag. $=1, W_{\text {in }}$ gain $=1$ ), they required considerable input weights changes (Supplementary Fig. 4c) to generate similarly-sized postsynaptic current changes as fully plastic networks (Supplementary Fig. 4e). Again, this was due to an asymmetry in the input and network unit firing rate magnitudes (Supplementary Fig. 4 d ) : the network unit firing rates further increased in response to the elevated input unit firing rates, because the network units were receiving larger currents from the input units.

Finally, we reasoned that this increase in the network unit firing rate magnitudes could be avoided by additionally scaling down the magnitude of the input weights. This would both generate similarly-sized efferent currents from the input units as in our original networks, and facilitate a stronger influence of input weight changes on the postsynaptic current changes due to the elevated input unit firing rates. We tested this in networks with both a tenfold increase in the input unit firing rates and a 20-fold decrease in the initial input weights (i.e. input weights of the naive network). We now found that networks with frozen recurrent weights exhibited learning efficiency asymptotes that were comparable to their fully plastic counterparts (Supplementary Fig. 4b, Input Act. Mag. $=10, W_{\text {in }}$ gain $\left.=0.05\right)$. These networks produced input weight changes of comparable magnitude to the weights changes in fully plastic networks (Supplementary Fig. 4 4 c), while also producing postsynaptic current changes of comparable magnitude (Supplementary Fig. 4e). This was because input and network unit firing rates were comparable in magnitude (Supplementary Fig. 4 $\mathrm{d}$ ). Taken together, these analyses demonstrate that fully plastic networks learn new problems largely via recurrent connection weight changes because it is generally more efficient to do so. However, recurrent weight changes are not necessary for efficient learning: Network regimes exist wherein learning via input weight changes is equally efficient. 


\subsection{Plasticity alters stimulus representations far more than de- cision representations}

We have demonstrated that the model learns a problem when each sample stimulus elicits decision and choice representations that appropriately direct the desired output response, and that this is achieved by the preferential engagement of plasticity in the network's recurrent connections. But does this process also enlist and alter the stimulus representations, and if so, to what end? Measurements showed that both the decision and the stimulus representations developed sizeable changes after learning (Supplementary Fig. 6 b). To test the utility of the changes in the stimulus representations, we simulated trials in a network that had learned a problem, while clamping its stimulus representations at their pre-learning values (see Methods), and measuring the effect of this intervention on the decision representations. If the learned decision representations evolve independently of the stimulus representations, they should remain largely unaltered. Instead, we found that the decision representations experienced large deviations (Supplementary Fig. 6c). Similarly, clamping the decision representations at their prelearning values produced large deviations in the stimulus representations. This shows that the stimulus and decision representations sustain strongly recurrent interactions, and that changes in the stimulus representations are necessary both to remap sensory inputs onto the appropriate decision manifold and to maintain these decision representations throughout the trial.

We also examined whether the decision and stimulus representations mutually influence each other's WdVFC. Specifically, how is the WdVFC modulated by pre-synaptic population activity in the stimulus versus decision subspaces? And to what extent does the resulting WdVFC alter subsequent stimulus versus decision representations? In figure 6 $\mathrm{c}$, we show that the WdVFC is primarily modulated by pre-synaptic population activity in the decision subspace, i.e. the decision representations predominantly scaffold the WdVFC. Moreover, this decision- and weight-driven vector field change is primarily responsible for learning - reversing it reduced output accuracy almost to chance levels, while reversing the stimulus- and weight-driven vector field change had a much weaker 
effect (Supplementary Fig. 61). Finally, a comparison of the overlap of the WdVFC with the stimulus versus decision subspaces showed that weight changes mostly alter stimulus representations (Supplementary Fig. 6e).

These results suggest that reciprocal interactions between the stimulus and decision representations play a key role not only in decision making and working memory maintenance of these decisions, but also in learning the two mappings in each problem. They further demonstrate that the decision representations scaffolds the WdVFC, and that the WdVFC largely prevents uncharacteristic changes to the existing stimulus representations, a finding that is consistent with our results in figure 5 and supplementary figure $7 a$. 


\section{Figures}

a

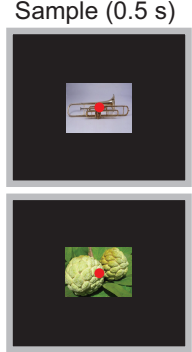

C

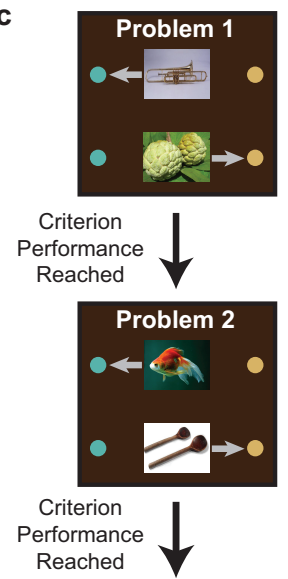

Delay (1 s) Response (0.5 s)
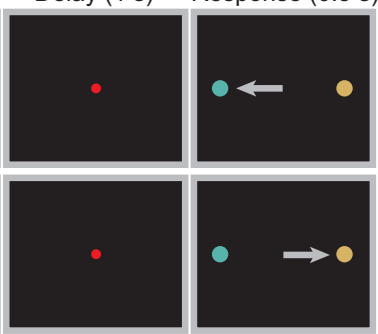

d

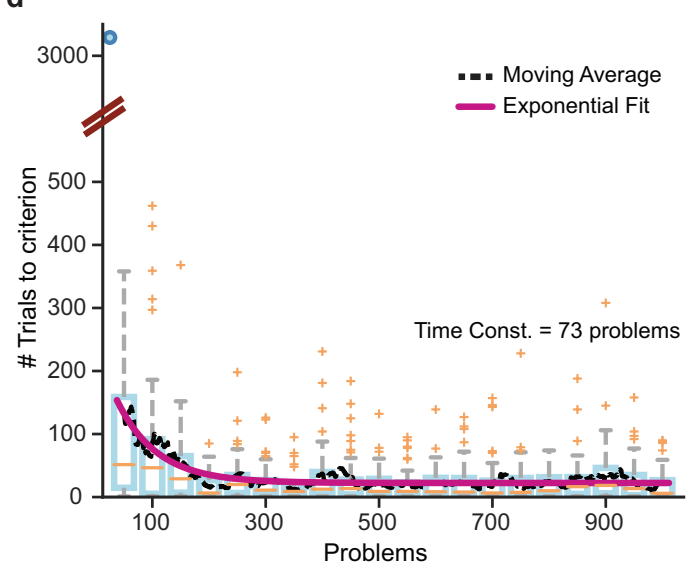

b
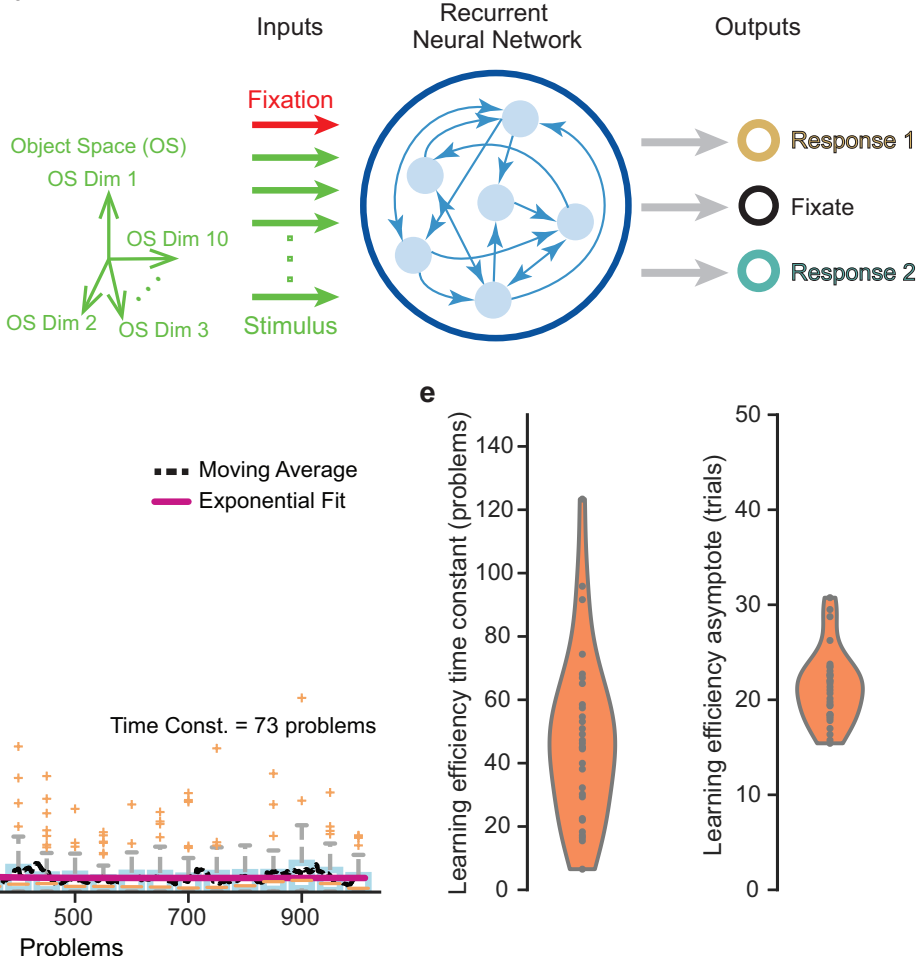

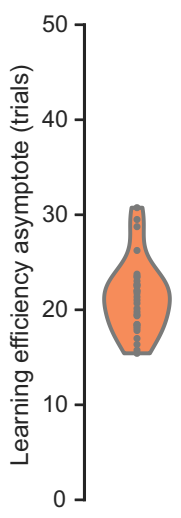

Figure 1: Recurrent neural networks trained on a series of delayed sensorimotor association problems learn-to-learn. a. An example problem illustrating the structure of the delayed sensorimotor association task. The model must learn to associate each of two sensory stimuli (e.g. images) with a corresponding motor response (e.g. a saccade). Targets are colored to emphasize the distinction between response choices, and not to indicate that the response targets are colored. $\boldsymbol{b}$. RNN model trained to perform the task. It is composed of recurrently connected rate units that receive a fixation stimulus and features of the sample sensory stimulus as inputs, and reports its response choices via output units corresponding to fixation, motor response choice 1 (brown), or motor response choice 2 (teal). c. Learning-toLearn training protocol. The model is trained on a series of sensorimotor association problems, each with randomly chosen sample stimulus pair. It is transitioned to a new problem once it reaches criterion performance on the current problem. $\boldsymbol{d}$. Learning efficiency, measured as the number of trials to criterion performance on a problem, over a series of 1000 problems learned by a network. Box plots summarize the learning efficiency in groups of 50 consecutive problems. The number of trials to criterion on a problem decreases with the number of previously learned 
bioRxiv preprint doi: https://doi.org/10.1101/2021.09.02 455707: this version posted September 2, 2021. The copyright holder for this preprint (which was not certified by peer review) is the author/funder, who has granted bioRxiv a license to display the preprint in perpetuity. It is made available under aCC-BY-NC 4.0 International license.

1331

problems. This is characterized by a decaying exponential function and demonstrates the model's ability to learn-to-learn. e. 30 RNNs with different initial conditions exhibit robust learningto-learn, as indicated by the time-constants (left) and asymptotes (right) of the exponential fits to their learning efficiency over a series of 1000 problems. 
a
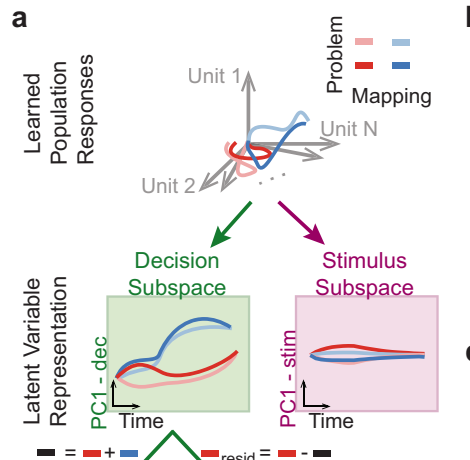

Mean

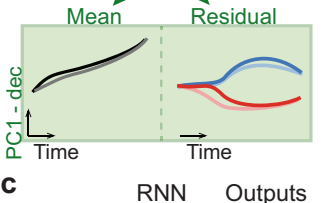

C

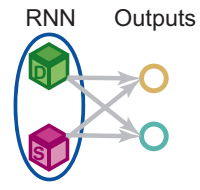

b

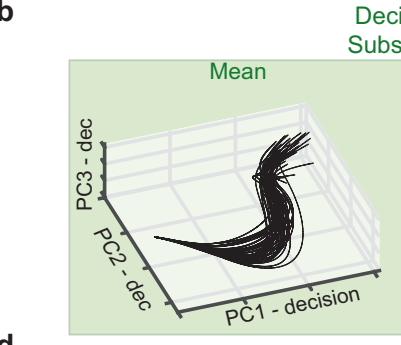

Decision

bspace
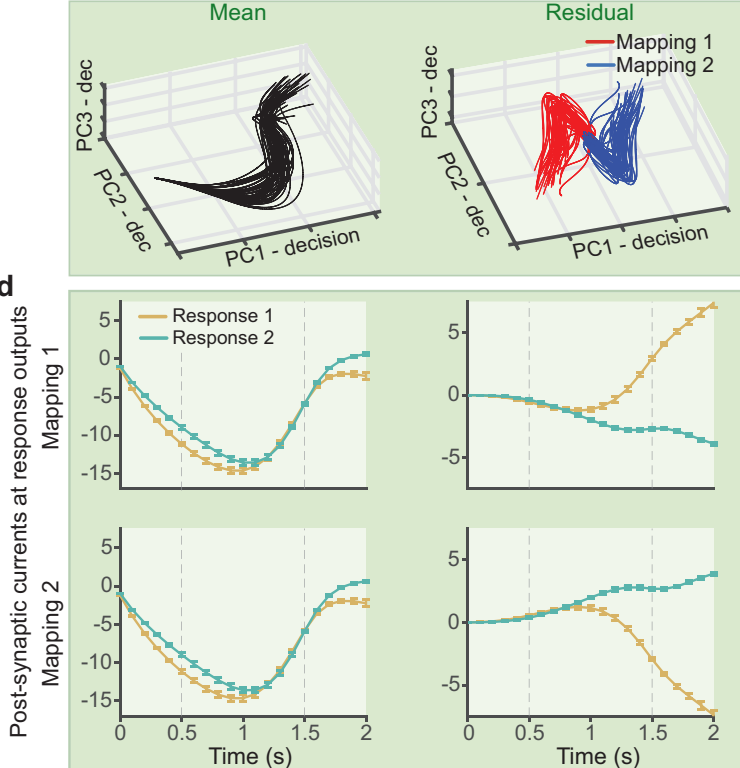

Stimulus

Subspace
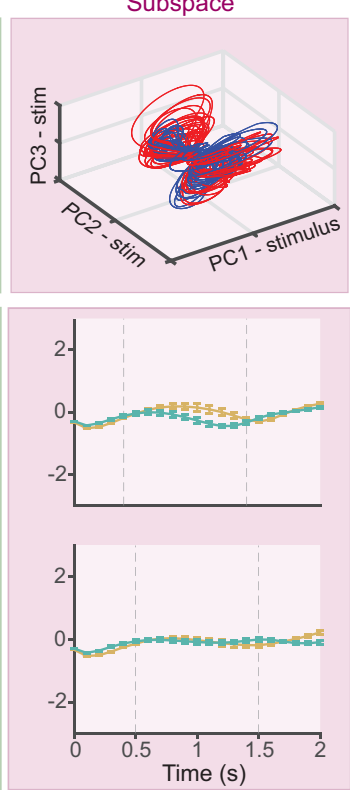

Figure 2: Neural representations of decision and choice are shared across prob-

lems. a. Schematic of the demixing procedure that identifies shared versus problem-dependent components of the neural representations. Population trajectories for the two mappings in 50 consecutively learned problems (illustrated for 2 problems, for clarity) are decomposed into components within a decision subspace which are shared by trajectories that map their respective sample stimuli onto a common response choice, and problem-dependent components embedded in a stimulus subspace. The shared decision representations are further decomposed into their mean and residual components for each problem. $\boldsymbol{b}$. Decomposed representations for problems 1-50, presented in the first 3 principal components of their respective subspaces. $c$. Schematic illustrating that the component representations collectively drive the response choice outputs. $\boldsymbol{d}$. The net current from the mean (left) and residual (center) decision representations, and the stimulus representations (right), to response 1 (brown) and response 2 (teal) outputs, in mapping 1 (top) and mapping 2 (bottom) trials. The mean decision components inhibit motor responses during the sample and delay epochs, and the residual decision components drive the correct response while inhibiting the incorrect one. Dashed vertical lines indicate the end of the sample and delay epochs. Plots show mean of the net currents across the 50 problems, and error bars indicate their standard errors. 

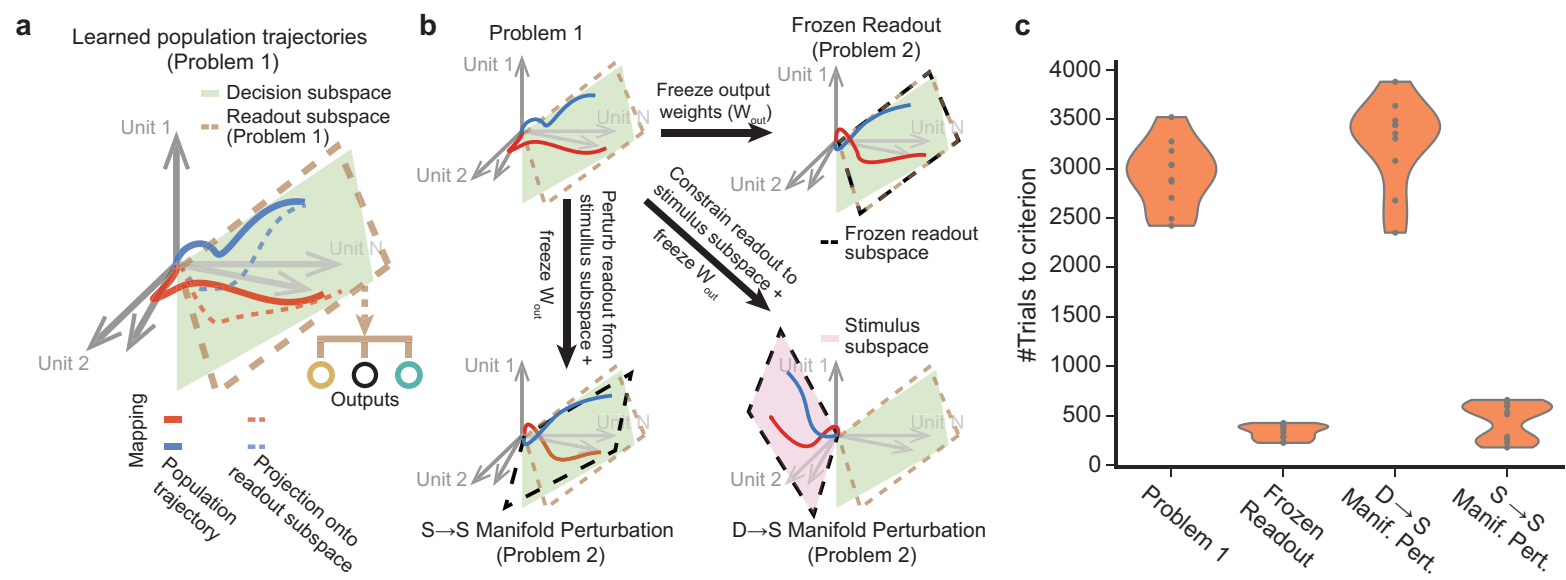

Figure 3: Manifold perturbations reveal that the reuse of the schematic decision manifold facilitates learning. a. Output responses are readout from a subspace of the population state space, which is spanned by the network's output weights. Overlap between this readout subspace and the decision subspace enables the control of output responses by the decision representations. $\boldsymbol{b}$. Illustration of the manifold perturbation interventions that assess the role of decision manifold reuse in learning. A network is trained on a single problem to establish its decision and readout subspaces (top left). It is then trained on a second problem (i) while its output weights are frozen (frozen readout, top right), (ii) after perturbing and freezing its output weights such that its readout subspace only overlaps with its stimulus subspace $(D \rightarrow S$ manifold perturbation, bottom right), or (iii) after perturbing and freezing its output weights such that the overlap between its readout and stimulus subspaces is altered $(S \rightarrow S$ manifold perturbation, bottom left). c. The average learning efficiency on the second problem in each of the three conditions, compared to the learning efficiency on the first problem. The trials-tocriterion on the second problem is averaged over 50 independently chosen random perturbations. Plot shows the distribution of average learning efficiencies across 10 networks with different initial conditions. 
a

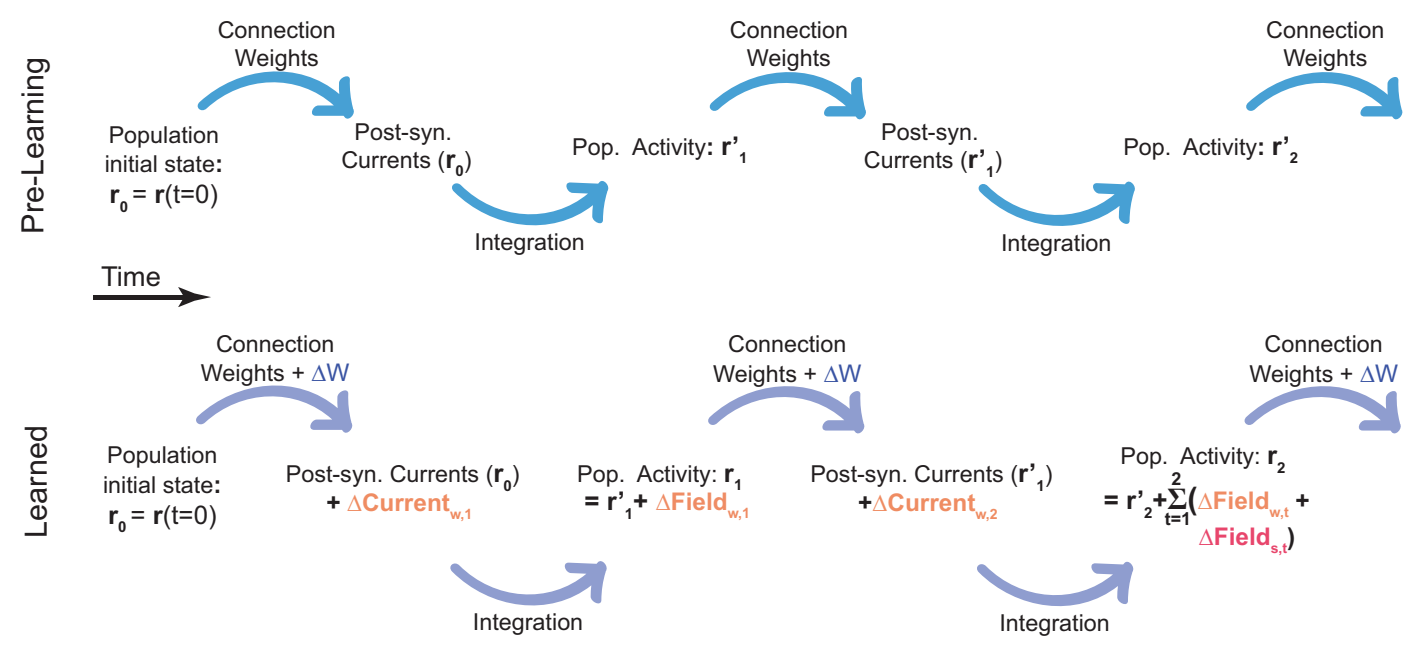

b Incremental activity change between Learned and Pre-Learning trajectories $(\Delta \mathbf{z})$ due to Vector field change $\left(\Delta\right.$ Field $_{w}, \Delta$ Field $_{\mathrm{s}}$ )

Population activity change

Between pre-learning

and learned trajectories $\left(\mathbb{Z}_{8}\right)$

$\mathbb{Z}_{\hat{t}}=r_{t}-r_{t}^{\prime}$

Increment between

consecutive time-steps $\left(\Delta z_{t}\right)$

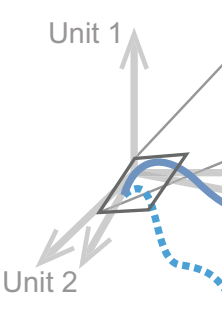

Pop. Trajectories

- $n=$ Pre-Learning

Learned

Figure 4: Learned trajectories emerge from vector field changes. a. Schematic describing the temporal evolution of population activity at the start (Pre-learning, top) and end (Learned, bottom) of a problem. The activity advances due to the integration of net postsynaptic currents, which depend on the activity levels of the network and input units and their efferent connection weights. Changes in population activity between the beginning and end of a problem are due to an accumulation of weight- $\left(\Delta \boldsymbol{F i e l d}_{\boldsymbol{w}}\right)$ and state-driven $\left(\Delta \boldsymbol{F i e l \boldsymbol { d } _ { \boldsymbol { s } } )}\right.$ vector field 
changes. $\boldsymbol{b}$. Illustration of the temporal evolution of pre-learning and learned trajectories in population state space (left). The instantaneous advance in population state determines a vector field that guides the evolution of population trajectories (top-center, blue arrows). The divergence of the learned trajectory from the pre-learning trajectory $\left(\boldsymbol{z}_{\boldsymbol{t}}\right.$, top-center, solid light green arrow) emerges from an accumulation of activity change increments throughout the trial ( $\Delta \boldsymbol{z}_{\boldsymbol{t}}$, top-center, dark green arrow). Each increment is the sum of the weight- and statedriven vector field changes (bottom-center, orange and pink arrows, respectively). The former is caused by plasticity-induced connection weight changes $(\Delta W)$, that alter the postsynaptic currents ( $\Delta$ Current $\left.\boldsymbol{w}_{\boldsymbol{w}}\right)$, thereby altering subsequent activity (top-right, and bottom panel of a). The latter is caused by state-dependent differences in the pre-learning vector field at learned and pre-learning population states (bottom-right, solid light blue arrows at $\boldsymbol{r}_{\mathbf{1}}$ and $\boldsymbol{r}_{\mathbf{1}}^{\prime}$, respectively). Dashed arrows represent vectors that have been displaced to clearly illustrate vector differences. In each illustration, irrelevant details are faded out to highlight relevant concepts. 
b

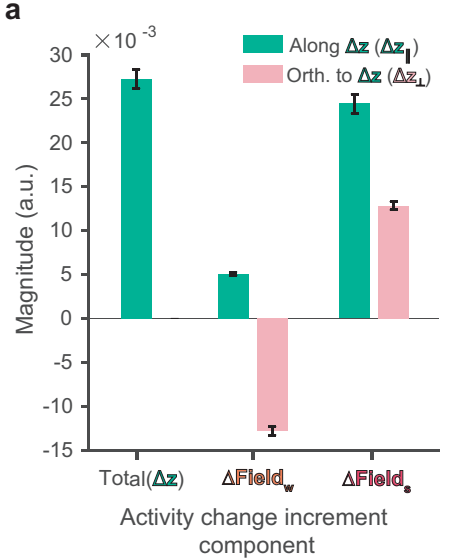

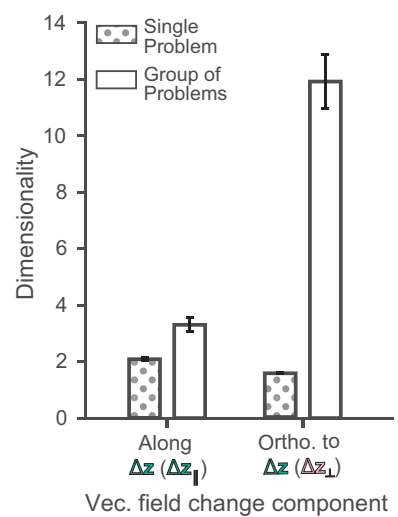

Figure 5: Weight- and state-driven vector field changes differentially contribute to population activity changes. a. Magnitude ( $L^{2}$-norm) of the population activity change increments, and its vector field change constituents (weight- and state-driven vector field changes). The magnitudes of the vector field changes are decomposed (by vector projection, see Methods) into components in the direction of (dark green), and orthogonal (light pink) to, the activity change increments. Measurements shown are the temporal mean of the magnitudes over the trial duration, averaged over both mappings of problems 2-51. b. Dimensionality of the vector field change components on single problems (averaged over problems 2-51) and for a group of 50 problems (problems 2-51). All bars represent the mean across 10 networks with different initial conditions, and error bars indicate their standard errors. 
a

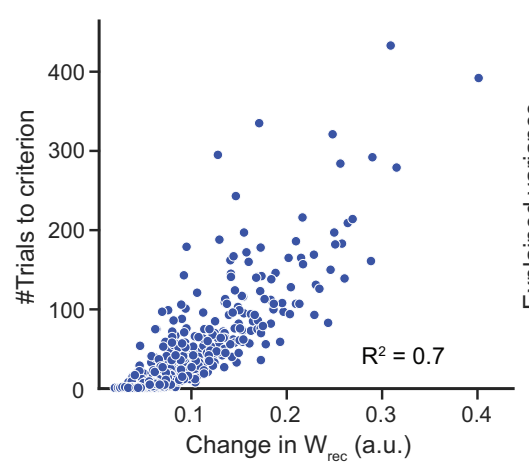

b

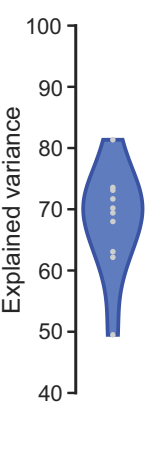

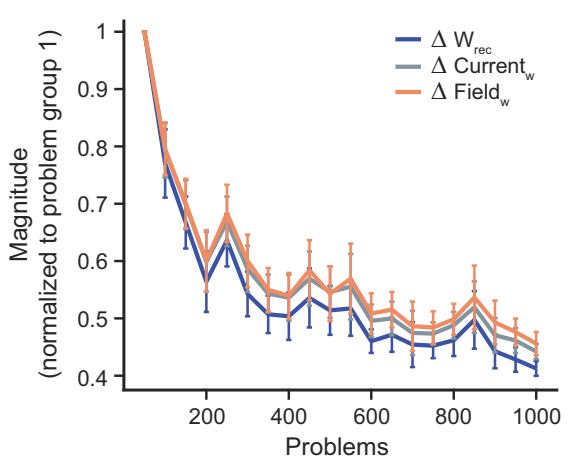

C

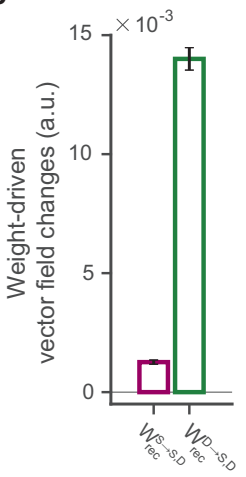

Figure 6: The magnitude of recurrent weight changes explains both the magnitude of the WdVFC and the number of trials to learn a problem. a. The magnitude of the plasticity-induced recurrent connection weight changes explains a majority of the variance in the number of trials to learn problems (left). This relationship was robustly observed across ten networks with different initial conditions (right). $\boldsymbol{b}$. The magnitude of recurrent weight (blue), postsynaptic current (gray) and weight-driven vector field (orange) changes, averaged in groups of 50 non-overlapping and consecutively learned problems. Each quantity has been normalized by its corresponding value for the first problem group. All quantities decrease exponentially with the number of previously learned problems. c. Approximate contribution of presynaptic population activity in the stimulus versus decision subspace to the weight-driven vector field change, averaged over problems 2-51. The magnitudes ( $L^{2}$-norm) of the change in the postsynaptic currents and vector field represent their temporal mean over the entire trial duration, averaged over both mappings in each problem. The magnitude of recurrent weight changes was measured by their Frobenius norm. Plots (b-c) reflects mean values over 10 networks with different initial conditions, and the error bars indicate their standard errors. 
a

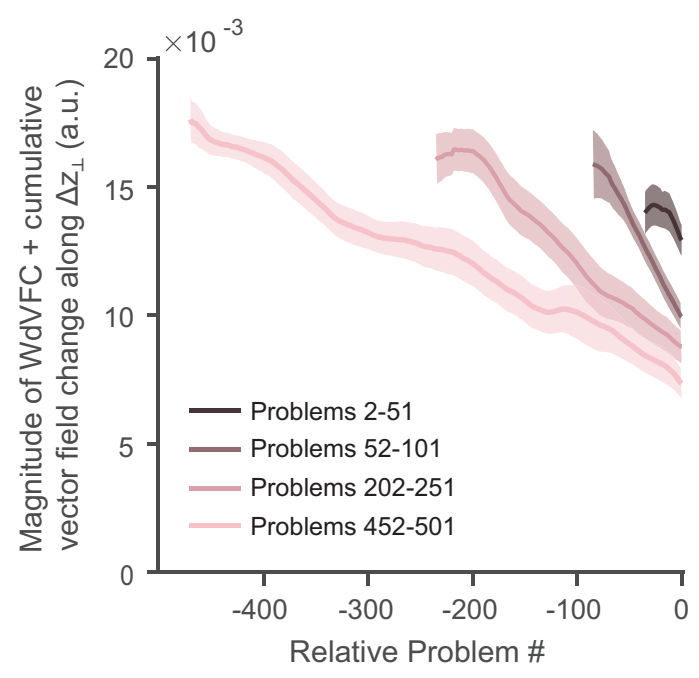

C

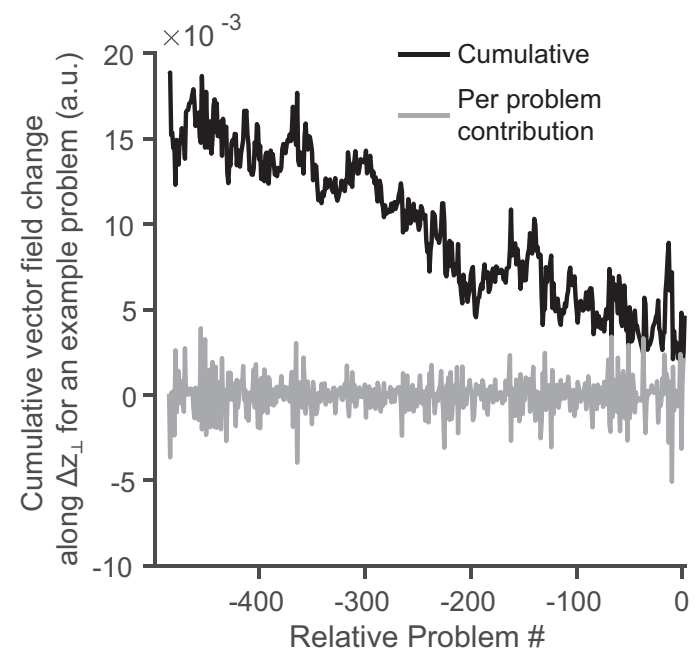

b

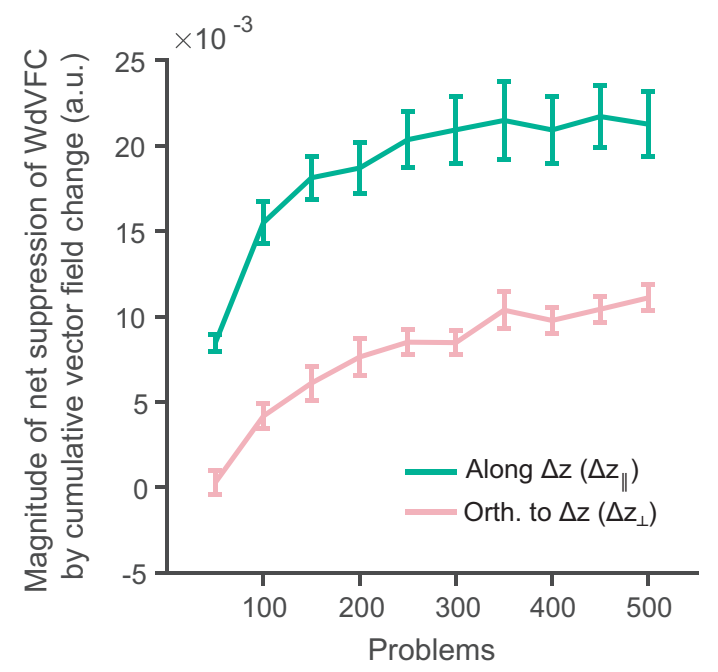

d

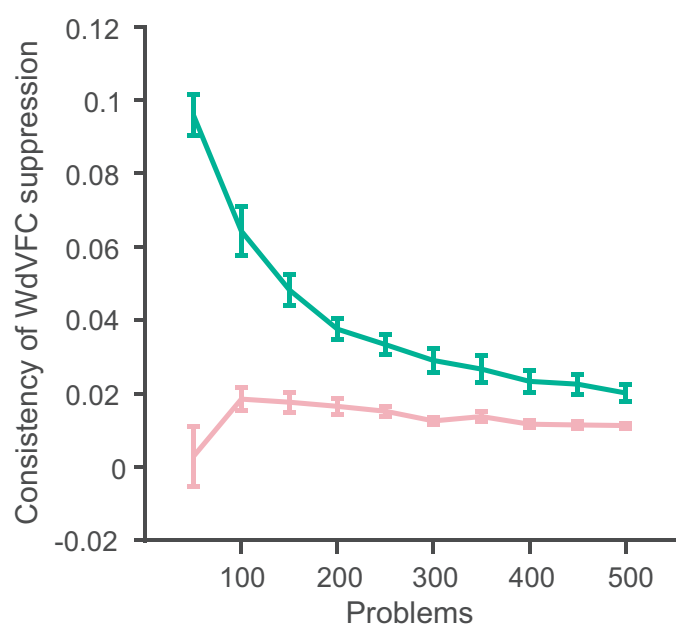

Figure 7: Accumulation of weight changes progressively improves invariance of existing representations to learning. a. Magnitude of vector field change along the learned trajectory for a problem $p$ due to the accumulation of $(i)$ the weight changes in problem $p\left(W^{p}-W^{p-1}\right.$, relative problem $=0$; weight-driven vector field change), and (ii) weight changes in each of the earlier problems, proceeding backwards to problem $2\left(W^{p}-W^{p-k-1}\right.$ for $1 \leq k \leq p-2$, relative problem $-k$; cumulative vector field change contributions). The curve for each problem measures the magnitude of change in the direction of its orthogonal WdVFC component, smoothed with a 30-problem moving average filter. Plot summarizes the measurements for problems in 4 problem groups at different stages of learning-to-learn, and 
demonstrates the suppressive effect of the cumulative vector field change (CVFC) at each stage. b. Magnitude of net suppression for each problem $p$, due to the net weight changes between the start of problems 2 and $p\left(W^{p-1}-W^{1}\right)$, summarized in 50-problem groups. $\boldsymbol{c}$. Magnitudes of the CVFC and CVFC contributions by individual problems along an example problem's orthogonal WdVFC component. d. Ratio of the net suppression magnitude to the sum of magnitudes of the CVFC contributions, summarized as in (b). This measures how consistently suppressive the CVFC contributions for a problem are. Measures in $(b, d)$ are presented separately for vector field changes along the parallel (green) and orthogonal (pink) WdVFC components. Magnitudes shown are the temporal mean of the unsigned $\left(L^{1}\right.$-norm; a) and signed $(b, c)$ projections onto the parallel / orthogonal WdVFC components, averaged over both mappings in a problem. Plots in ( $a, b$ and $d$ ) reflect mean values over 10 networks with different initial conditions, and shading/error bars indicate standard errors. 


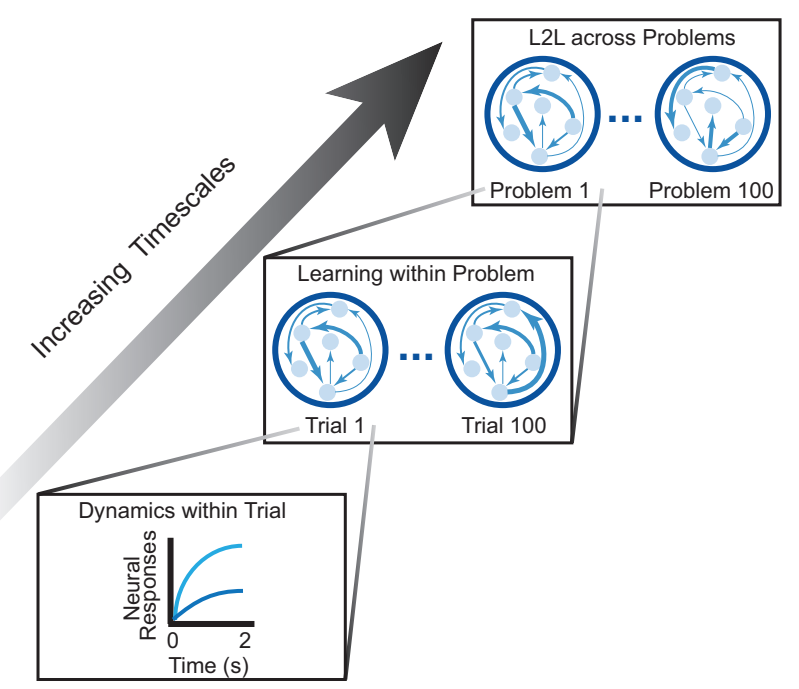

Figure 8: Learning-to-learn is a process with three timescales. The fastest timescale (bottom) governs the neural dynamics within a trial which drive output responses. The intermediate timescale (middle) governs the learning dynamics across trials within a problem; it ultimately produces the requisite weight-driven vector field change which results in problem being learned. The slowest timescale (top) governs the dynamics of learning-to-learn across problems; it ultimately improves the invariance of existing representations to learning new problems which results in asymptotic learning efficiency. 


\section{Supplementary Figures}
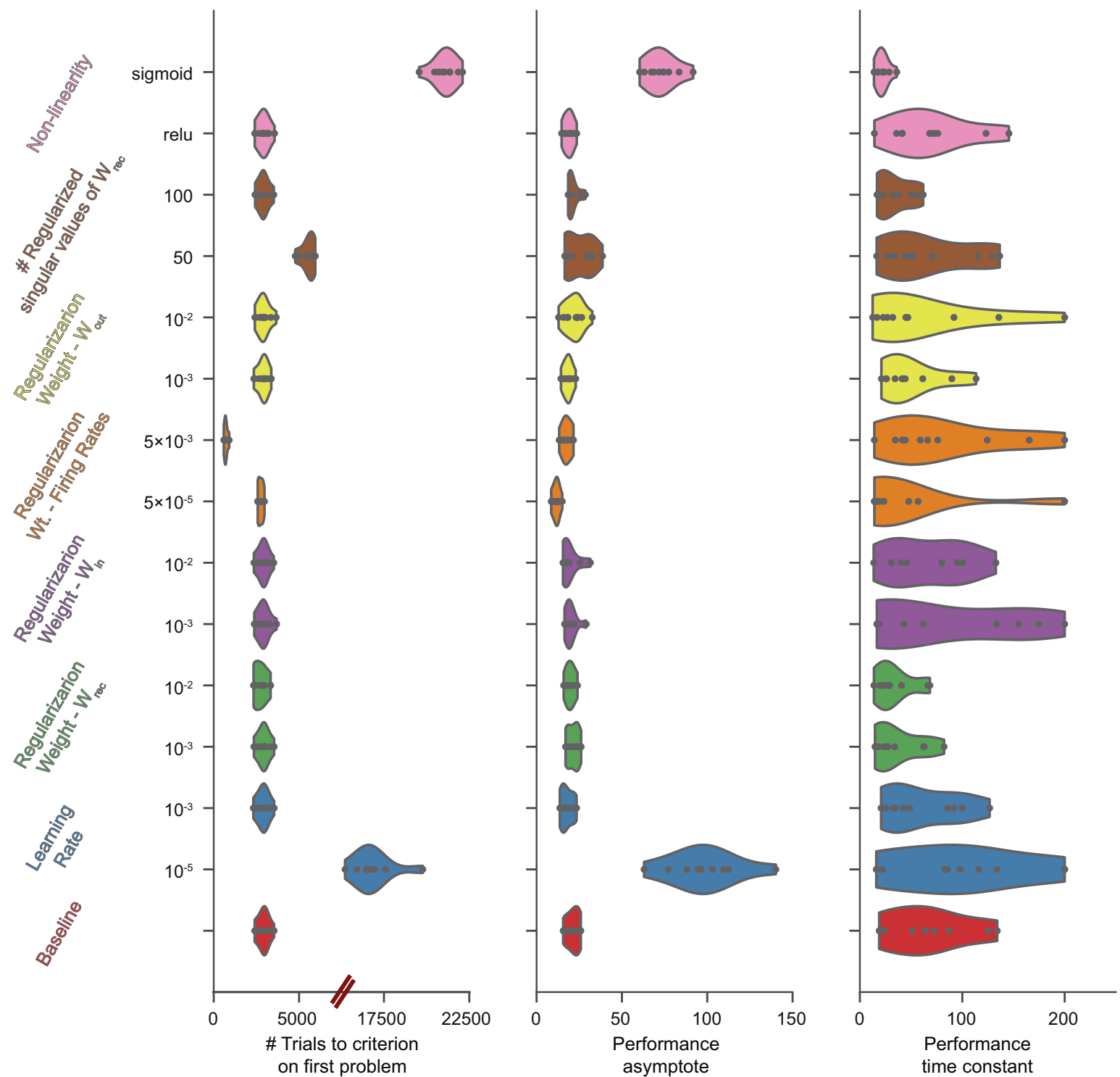

Supplementary Figure 1: Learning-to-learn is robustly observed across a range of hyper-parmeter settings. Problem 1 learning efficiency (left), learning efficiency asymptotes (middle), and learning efficiency time constants (right), of networks trained with different learning rates, recurrent $\left(\beta_{W_{\text {rec }}}\right)$, input $\left(\beta_{W_{i n}}\right)$ and output $\left(\beta_{W_{\text {out }}}\right)$ weight regularization levels, numbers of recurrent weight matrix singular values $(k)$ that are regularized, firing rate regularization levels $\left(\beta_{r}\right)$, and $f$-I transfer functions. Performance measures are presented in comparison to the baseline networks discussed in the main text. The regularization hyperparameters and learning rates spanned 2 orders of magnitude. 10 networks were trained per 
bioRxiv preprint doi: https://doi.org/10.1101/2021.09.02 455707· this version posted September 2, 2021. The copyright holder for this preprint (which was not certified by peer review) is the author/funder, who has granted bioRxiv a license to display the preprint in perpetuity. It is made available under aCC-BY-NC 4.0 International license.

1465

hyper-parameter setting. Networks with sigmoid f-I transfer functions were trained with input unit firing rates scaled up by a factor of 10. Networks at the slowest learning rate and those with a sigmoid f-I transfer functions exhibited slower learning. However, all networks demonstrated learning-to-learn. 
a

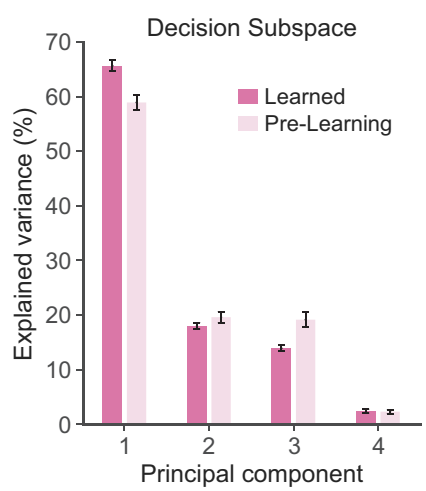

b

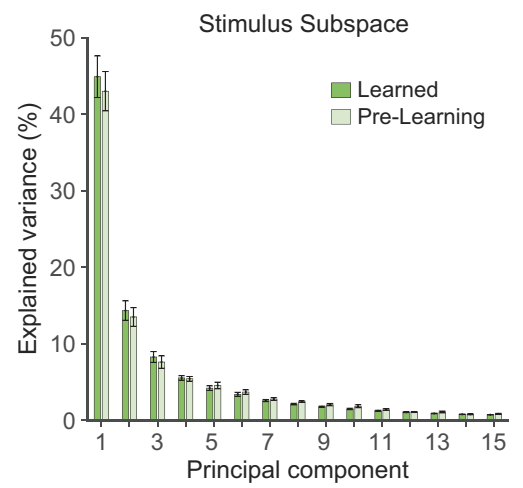

c

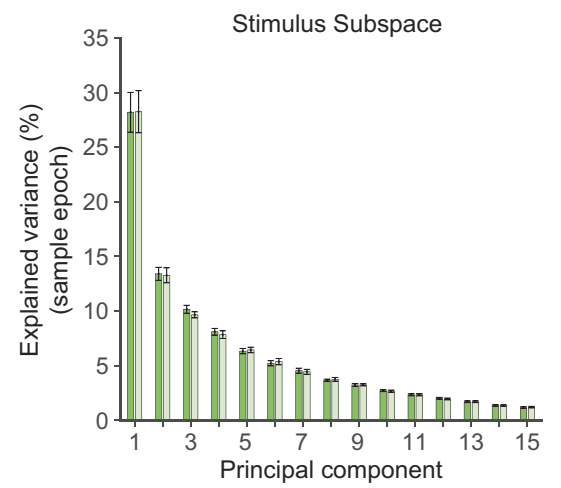

Supplementary Figure 2: Intrinsic structure of the learned neural representations is also recruited in response to novel sample stimuli. a. Comparison of the variance in pre-learning and learned population activity that is explained by each principal component of the decision subspace. The decision subspace and its principal components were computed from the learned population activity across 50 consecutive problems. The variance in both the pre-learning and learned activity was measured along these principal components. $\boldsymbol{b}$-c. Comparison of the variance in pre-learning and learned population activity that is explained by the first 15 principal component of the stimulus subspace, measured across the entire trial duration (b) and across the sample epoch of the trials only (c). The structure underlying learned neural representations is recruited even at the start of each problem, when the sample stimuli presented to the network are novel. The first problem was excluded from the pre-learning variance measurements, as the decision and stimulus representations develop only after the first problem is learned. Bars show mean variance explained are across 10 networks with different initial conditions, and error bars indicate standard errors of the mean. 
b

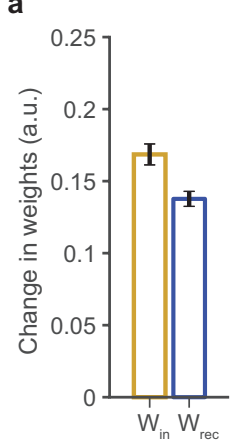

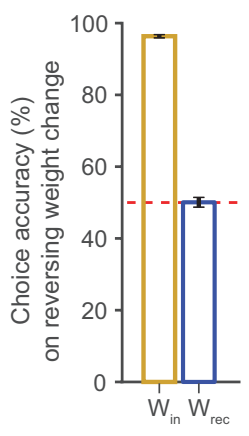

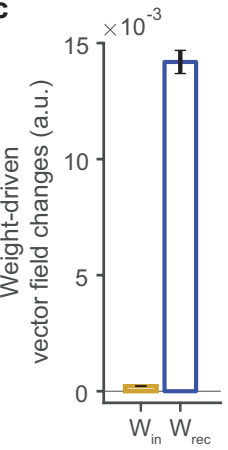

Supplementary Figure 3: Learning largely relies on changes in the recurrent connection weights. a. Magnitude of the recurrent and input connection weight changes, measured by their Frobenius norms. $\boldsymbol{b}$. Output accuracy when either the input or recurrent connection weight changes are reversed. c. Approximate magnitude of the weight-driven vector field change due recurrent and input connection weight changes. Magnitudes shown are the temporal mean of the $L^{2}$-norm of the corresponding vector quantities over the entire trial duration, and averaged over both mappings in a problem. All measures presented are averages across problems 2 thru 51. All bars represent the mean across 10 networks with different initial conditions, and error bars indicate their standard errors. 

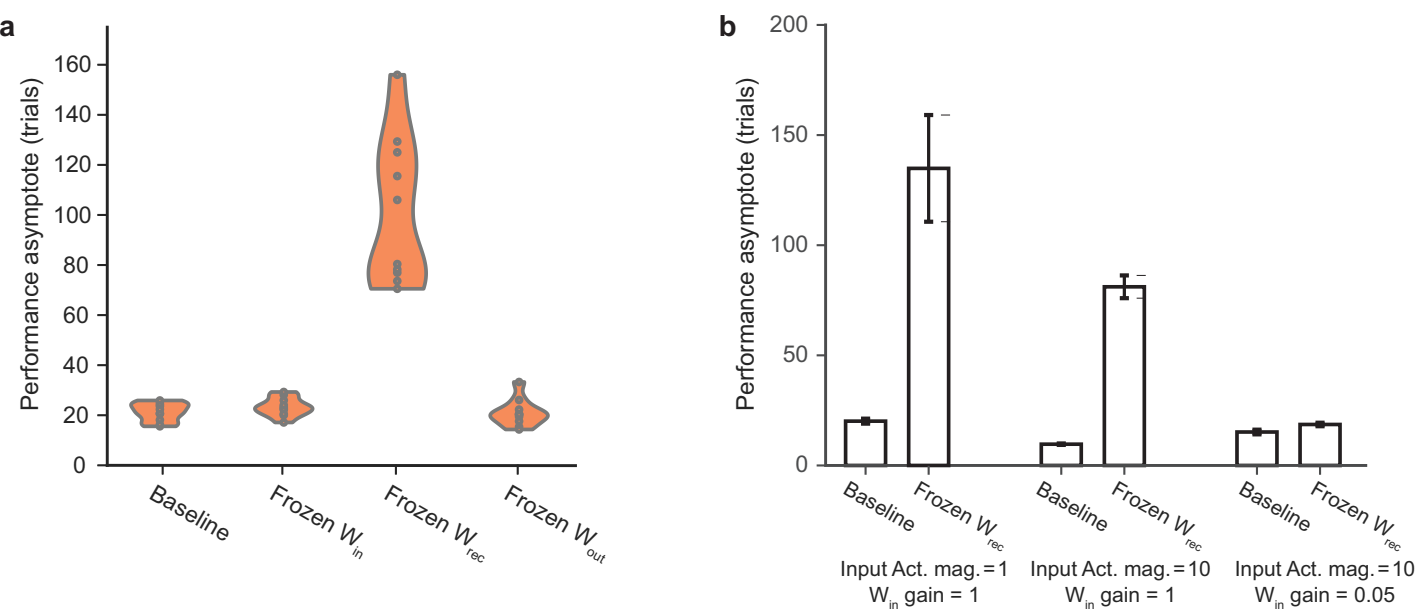

1493

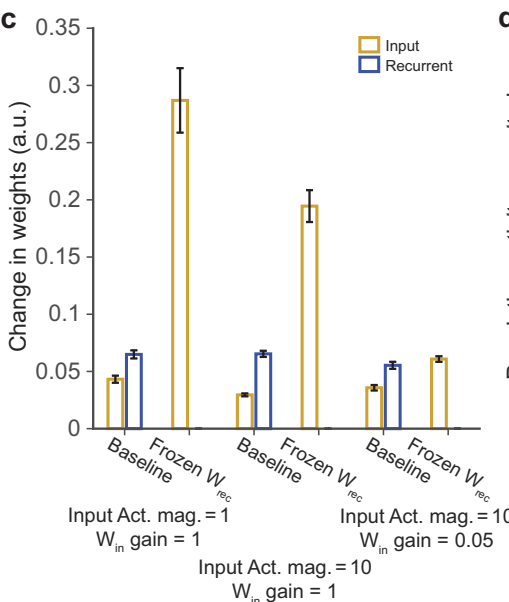

$W_{\text {in }}$ gain $=1$

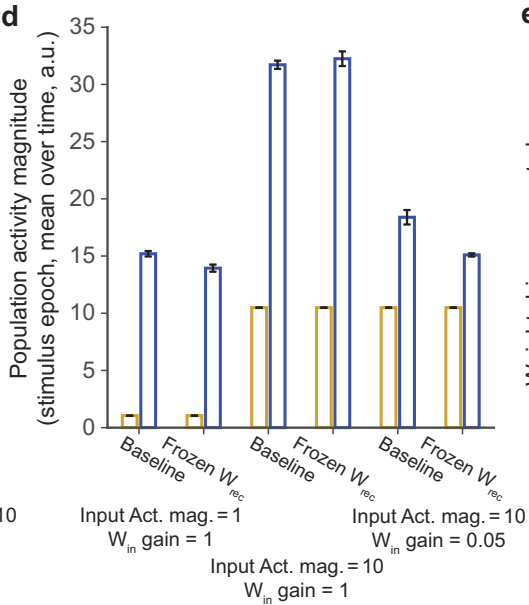

$W_{\text {in }}$ gain $=1$

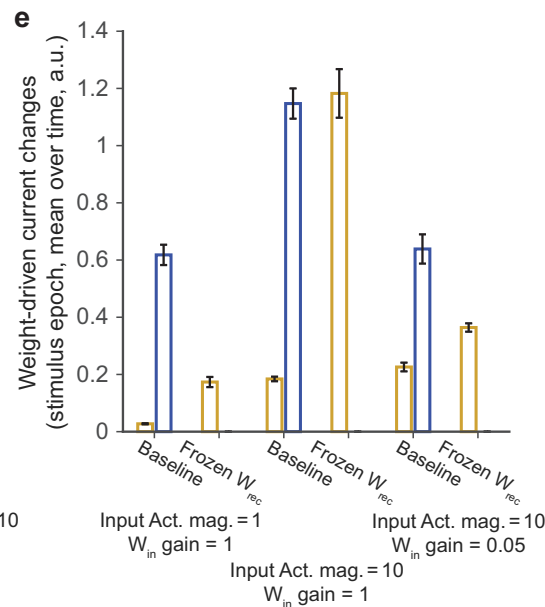

Supplementary Figure 4: Plasticity of recurrent connection weights results in, but is not necessary for, efficient learning. a. Comparison of the learning-to-learn asymptotes of fully plastic networks (Baseline), and those trained with frozen input, recurrent or output weights. Weights were frozen after the first problem was learned, to ensure the emergence of the decision and stimulus manifolds. $\boldsymbol{b}$-e. Comparison of fully plastic networks (baseline) and those with frozen recurrent weights in three network regimes that differed in the magnitude of the input unit firing rates (Input Act. mag.) and the relative strengths of the initial input connection weights ( $W_{\text {in }}$ gain). Comparison of the learning-to-learn asymptotes (b), magnitude (Frobenius norm) of input and recurrent weights changes (c), magnitude ( $L^{2}$ norm) of input and network unit firing rates (d), and magnitude ( $L^{2}$ norm) of the input and recurrent postsynaptic current changes (e). Quantities in (d-e) are temporal means over the sample epoch of the trials. Quantities in (c-e) are averages across problems 951-1000. All bars 
bioRxiv preprint doi: https://doi.org/10.1101/2021.09.02 455707; this version posted September 2, 2021. The copyright holder for this preprint (which was not certified by peer review) is the author/funder, who has granted bioRxiv a license to display the preprint in perpetuity. It is made available under aCC-BY-NC 4.0 International license.

1508

represent means across 10 networks with different initial conditions, and error bars indicate their standard errors. 
1509

a

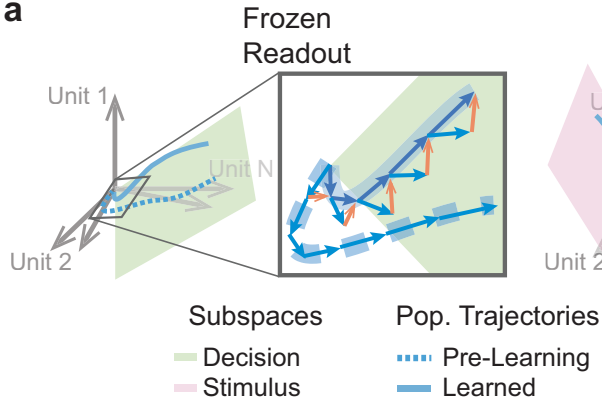

Frozen

- Decision

- Learned

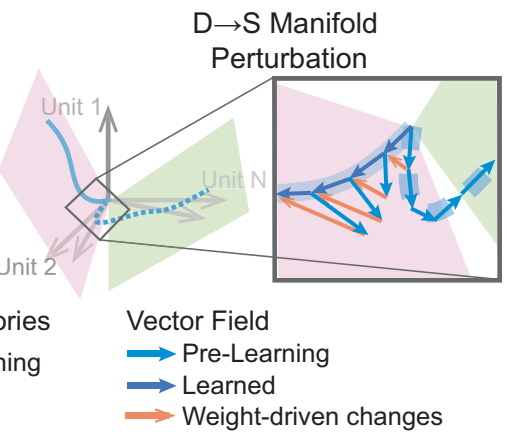

$\rightarrow$ S Manifold

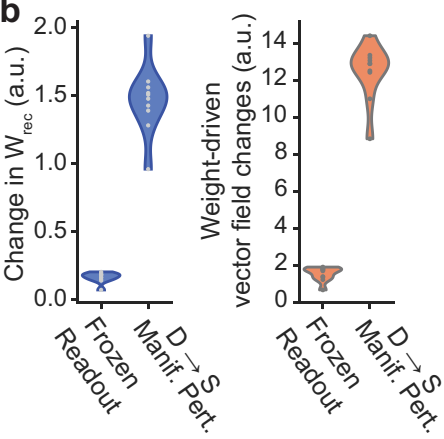

1510

Supplementary Figure 5: The pre-existing decision manifold provides a representational scaffold for the formation of learned trajectories in new problems.

a. Schematic illustrating pre-learning and learned population trajectories, together with the requisite weight-driven vector field change (orange arrows) in a network with frozen readouts (left) and a network with a $D \rightarrow S$ manifold perturbation (right). Vector field shaped by prior learning is approximately oriented to support the evolution of learned trajectories in networks with frozen readouts, but not in networks with $D \rightarrow S$ manifold perturbations. Therefore, the latter requires a substantial weight-driven vector field change. $\boldsymbol{b}$. Distribution of the magnitude of recurrent weight (left) and weight-driven vector field (right) changes required to learn the second problem, in the frozen readout and $D \rightarrow S$ manifold perturbation conditions, across 10 networks with different initial conditions. Smaller weight changes required by networks with frozen readouts makes them efficient learners. For each network, the measures were averaged over 50 perturbations, with new sample stimuli used each time. The magnitude $\left(L^{2}\right.$-norm) of the weight-driven vector field change represented is its temporal mean over the entire trial duration, averaged over both mappings in each problem. 
a

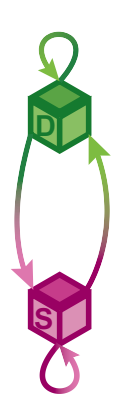

b

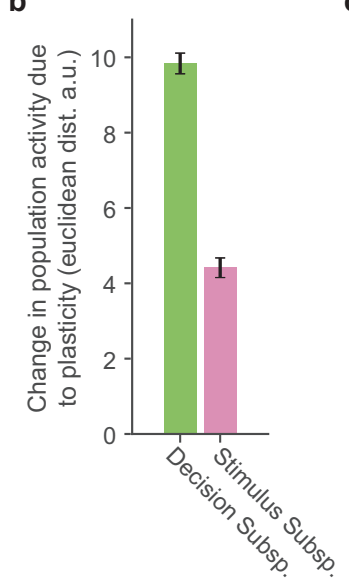

c

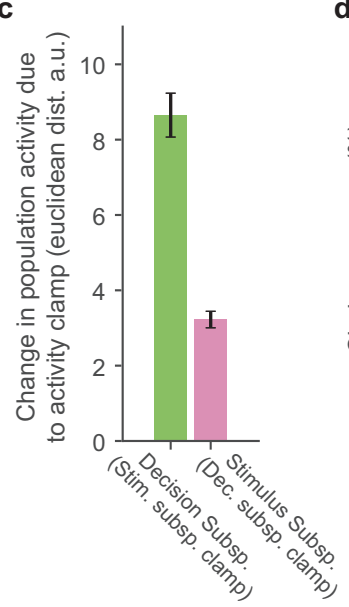

d

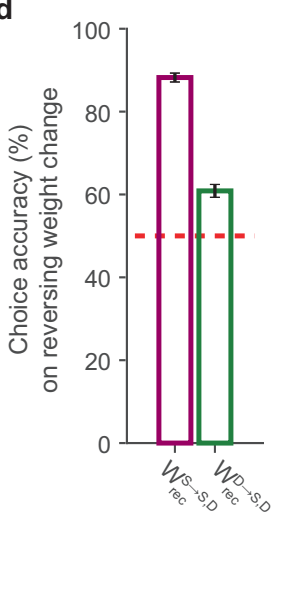

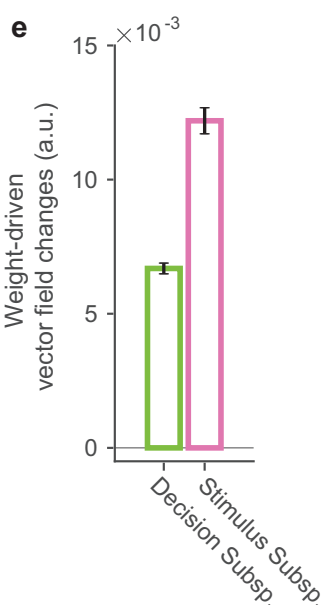

Supplementary Figure 6: Reciprocal interactions between stimulus and decision representations shape network dynamics and support learning. a. Schematic of recurrent interactions within and between stimulus (magenta) and decision (green) representations. Efferent (afferent) connections are represented in darker (lighter) colors. b. Euclidean distance between pre-learning and learned decision and stimulus representations. c. Euclidean distance between learned decision (stimulus) representations and those generated by the network when simulated with its stimulus (decision) representations clamped to their pre-learning values. $\boldsymbol{d}$. Output accuracy when the weight-driven current changes modulated by presynaptic population activity in the stimulus or decision subspace are reversed. e. Magnitude (L $L^{2}$-norm) of weightdriven vector field change within the stimulus and decision subspaces. The magnitudes and Euclidean distances represented are their temporal mean over the entire trial duration, averaged over both mappings in problems 2-51. All bars represent mean values over 10 networks with different initial conditions, and error bars indicate their standard errors. 
a

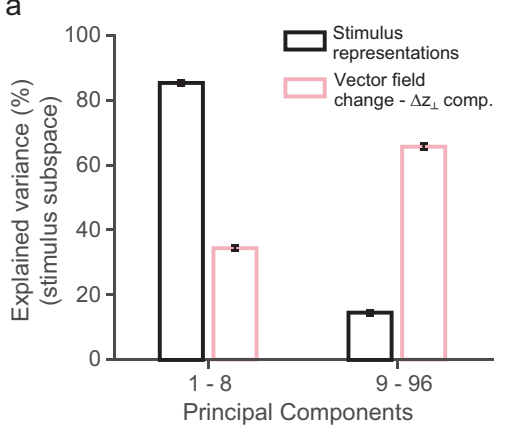

b

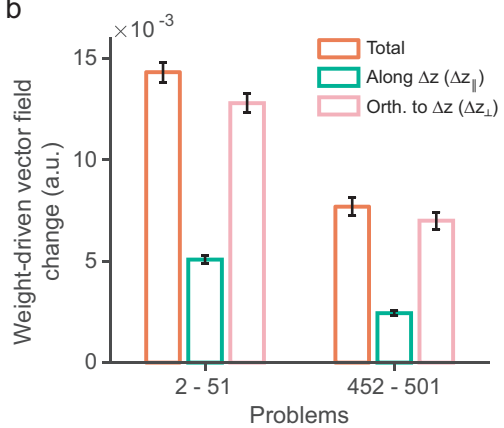

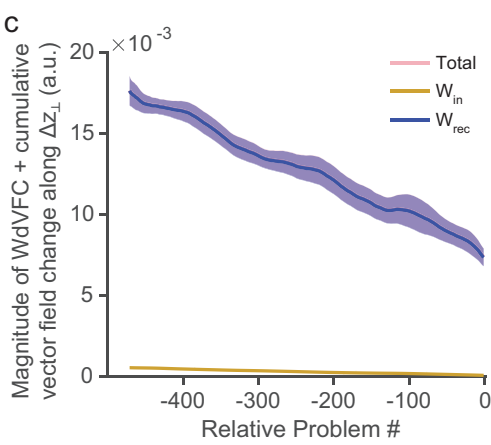

Supplementary Figure 7: Properties and contributions of the orthogonal components of the vector field change. a. Comparison of the percentage of variance in the stimulus representations (black) and the orthogonal components of the vector field change within the stimulus subspace (pink) explained by the first 8 and remaining principal components of the stimulus subspace. The orthogonal components largely lie off the stimulus manifold. $\boldsymbol{b}$. Magnitudes of the weight-driven vector field change (orange), and its components in the direction of the activity change increments (green) and orthogonal them (pink), for early and late learned problem groups. The orthogonal components dominate the total weight-driven vector field change across the learning-to-learn timecourse. c. Magnitude of vector field change along the learned trajectory for a problem $p$ due to the accumulation of (i) the weight changes in problem $p\left(W^{p}-W^{p-1}\right.$, relative problem $\left.=0\right)$, and (ii) weight changes in each of the earlier problems, proceeding backwards to problem $2\left(W^{p}-W^{p-k-1}\right.$ for $1 \leq k \leq p-2$, relative problem $-k)$. The curve for each problem measures the magnitude of change in the direction of its orthogonal WdVFC component, smoothed with a 30-problem moving average filter. Plot summarizes the measurements for problems at late-stage learning-to-learn (problems 452-501), and separately shows contributions of changes in input weights (yellow), recurrent weights (blue), and both (pink). The cumulative suppression of the weight-driven vector field change in the direction of its orthogonal component is almost entirely caused by an accumulation of recurrent weight changes. The magnitude ( $L^{2}$-norm) of the vector field change represented in $(b-c)$ is its temporal mean over the entire trial duration, averaged over both mappings of all the problems in the respective group of 50 problems. All plots represent mean values over 10 networks with different initial conditions, and shading/error bars indicate their standard errors. 

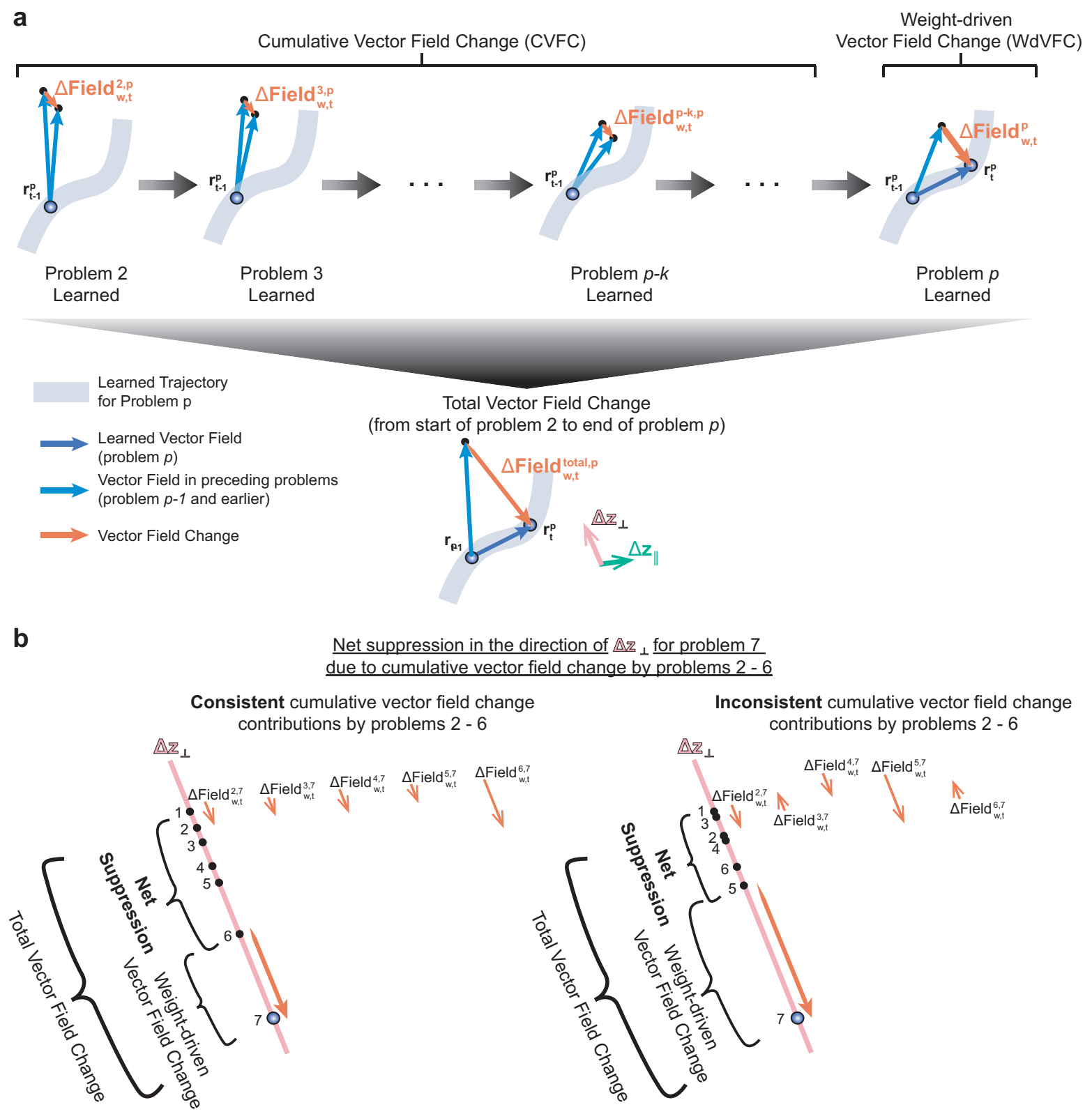

Similar-sized CVFC contributions in both conditions

Larger Net Suppression

1565

Smaller Net Suppression

Larger Weight-driven Vector Field Change

Supplementary Figure 8: Weight-driven vector field changes in earlier problems cumulatively suppress the weight-driven vector field change required to learn future problems. a. Schematic illustrating cumulative changes in the vector field along the learned 
trajectory for problem $p$ due to an accumulation of the weight changes elicited while learning the problems that precede it. This cumulative vector field change reduces the weight-driven vector field change required to support the evolution of problem p's learned trajectory. The total vector field change at problem $p$ measures the net effect of all the vector field changes along problem p's learned trajectory due to the weight changes between the start of problem 2 and the end of problem p. $\boldsymbol{b}$. Difference between consistently and inconsistently suppressive cumulative vector field change contributions illustrated along a problem's orthogonal vector field change component. Stronger consistency produces a larger net suppression, which reduces the requisite weight-driven vector field change by a larger amount. 
a

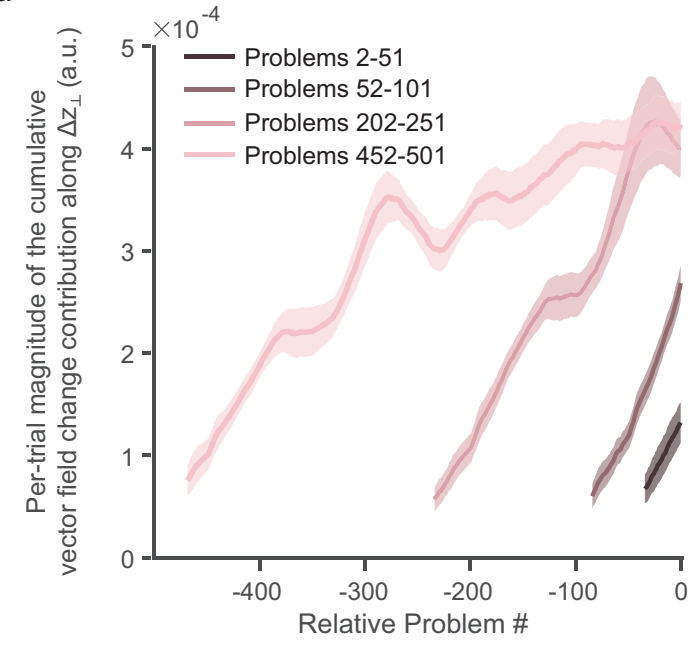

b

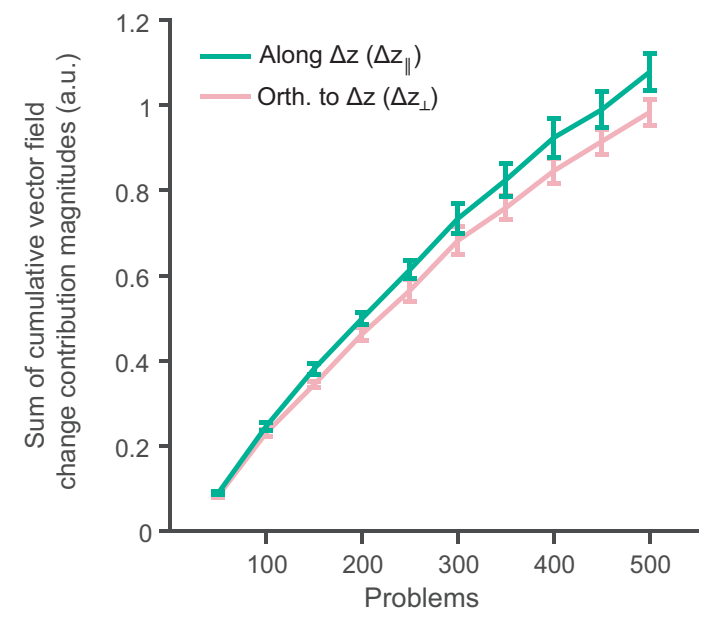

Supplementary Figure 9: Properties of vector field change along the learned trajectory for a problem due to weight changes in the preceding problems. a. Magnitude of per-trial cumulative vector field change (CVFC) contributions along the learned trajectory for a problem $p$ by the weight changes in each of the earlier problems, proceeding backwards to problem $2\left(W^{p}-W^{p-k-1}\right.$ for $1 \leq k \leq p-2$, relative problem $\left.-k\right)$. The curve for each problem measures the per-trial magnitude of change in the direction of its orthogonal WdVFC component, smoothed with a 30-problem moving average filter. Plot summarizes the measurements for problems in 4 problem groups at different stages of learning-to-learn. $\boldsymbol{b}$. Sum of the magnitudes of the CVFC contributions for each problem $p$ due to weight changes in problems 2 thru $p-1$. summarized in 50-problem groups. The measure is presented separately for the vector field change along the parallel (green) and orthogonal (pink) WdVFC components. Magnitudes shown in both plots are the temporal mean of the unsigned projections onto the parallel / orthogonal WdVFC components, averaged over both mappings in a problem. Plots reflect mean values over 10 networks with different initial conditions, and shading/error bars indicate standard errors. 